\title{
Holomorphy types and ideals of multilinear mappings
}

\author{
by \\ G. Botelho (Uberlândia), H.-A. Braunss (Potsdam), \\ H. Junek (Potsdam) and D. Pellegrino (Campina Grande)
}

\begin{abstract}
We explore a condition under which the ideal of polynomials generated by an ideal of multilinear mappings between Banach spaces is a global holomorphy type. After some examples and applications, this condition is studied in its own right. A final section provides applications to the ideals formed by multilinear mappings and polynomials which are absolutely $(p ; q)$-summing at every point.
\end{abstract}

Introduction. Special classes of homogeneous polynomials between Banach spaces have been studied by taking two different approaches. On the one hand, inspired by the dual theory of polynomials, L. Nachbin [19] introduced holomorphy types as classes of polynomials which are uniformly stable under differentiation. On the other hand, as a natural consequence of the successful theory of operator ideals, A. Pietsch [25] introduced the notion of ideals of multilinear mappings, which was immediately adapted to polynomials.

Both notions have been widely studied, holomorphy types as a branch of infinite-dimensional holomorphy (see the references cited in [12, p. 135]) and ideals of multilinear mappings/polynomials as a branch of Banach space theory (see $[3,9]$ and the references therein). Many outstanding examples, such as nuclear and compact polynomials, are simultaneously holomorphy types and ideals of polynomials. In this paper we try to give a unified treatment of the subject, exploring the interplay between these two notions, mainly trying to identify when an ideal of multilinear mappings generates a (global) holomorphy type. Recently, some particular ideals of polynomials have been proved to be global holomorphy types, for example: everywhere absolutely $(p ; q)$-summing polynomials (M. Matos [17]), strongly almost $q$-summing

2000 Mathematics Subject Classification: Primary 46G25, 46G20; Secondary 47B10.

Key words and phrases: holomorphy type, ideal of multilinear mappings.

Research of G. Botelho supported by Instituto do Milênio, AGIMB-IMPA and by FAPEMIG.

Research of D. Pellegrino supported by Instituto do Milênio, AGIMB-IMPA and by $\mathrm{CNPq} / \mathrm{FAPESQ}$. 
polynomials (D. Pellegrino [20]), fully (or multiple) summing polynomials (D. Pellegrino and M. Souza [23]) and mixing summing polynomials (M. Matos [18]).

In an attempt to treat this question in a systematic way, we first identify a condition under which a Banach ideal of multilinear mappings generates a global holomorphy type. This condition, which we call property (B), is applied to the two methods introduced by A. Pietsch in [25], namely the linearization and the factorization methods. Once the relevance of property (B) is established we study it in its own right and extend it to ideals of polynomials. In both the multilinear and polynomial cases we identify situations where having property (B) is equivalent to being (or generating) a global holomorphy type. Differences between the real and complex cases show that, though holomorphy types are more closely related for ideals of polynomials, property (B) is more appropriate for ideals of multilinear mappings. The paper ends with applications of the results obtained to the ideals formed by multilinear mappings and polynomials which are absolutely $(p ; q)$-summing at every point.

Some of the results, such as Theorems 3.2, 4.1 and 5.1, appeared, either exactly or at least in essence, in the second named author's thesis [6].

1. Definitions and notations. Throughout this paper $n$ is a positive integer, and $E_{1}, \ldots, E_{n}, E, F, G_{1}, \ldots, G_{n}$ and $G$ are Banach spaces over $\mathbb{K}=$ $\mathbb{R}$ or $\mathbb{C}$. $E^{\prime}$ is the dual space of $E$ and $B_{E}$ denotes the closed unit ball of $E$. The Banach space of all continuous $n$-linear mappings $A: E_{1} \times \cdots \times E_{n} \rightarrow F$ will be denoted by $\mathcal{L}\left(E_{1}, \ldots, E_{n} ; F\right)\left(\mathcal{L}\left({ }^{n} E ; F\right)\right.$ if $\left.E_{1}=\cdots=E_{n}=E\right)$, and the Banach space of all continuous $n$-homogeneous polynomials $P: E \rightarrow F$ by $\mathcal{P}\left({ }^{n} E ; F\right)$. If $F=\mathbb{K}$ we use the simplified notations $\mathcal{L}\left(E_{1}, \ldots, E_{n}\right), \mathcal{L}\left({ }^{n} E\right)$ and $\mathcal{P}\left({ }^{n} E\right)$. By $\check{P}$ we mean the unique continuous symmetric $n$-linear mapping associated to the polynomial $P$, and by $\widehat{A}$ the polynomial generated by a multilinear mapping $A$, that is, $\widehat{A}(x)=A(x, \ldots, x)$. For the general theory of multilinear mappings and homogeneous polynomials we refer to S. Dineen [12].

Definition 1.1. Although L. Nachbin [19] introduced the notion of holomorphy type between two fixed Banach spaces $E$ and $F$, all interesting examples work for every couple of Banach spaces. So, the following definition is quite natural. A global holomorphy type $\mathcal{P}_{H}$ is a class of continuous homogeneous polynomials between Banach spaces such that for every natural $n$ and any Banach spaces $E$ and $F$, the component

$$
\mathcal{P}_{H}\left({ }^{n} E ; F\right):=\mathcal{P}\left({ }^{n} E ; F\right) \cap \mathcal{P}_{H}
$$

is a linear subspace of $\mathcal{P}\left({ }^{n} E ; F\right)$ which is a Banach space when endowed with a norm denoted by $P \mapsto\|P\|_{H}$, and 
(i) $\mathcal{P}_{H}\left({ }^{0} E ; F\right)=F$, as a normed linear space for all $E$ and $F$,

(ii) there is $\sigma \geq 1$ such that for any Banach spaces $E$ and $F, n \in \mathbb{N}$, $k \leq n, a \in E$ and $P \in \mathcal{P}_{H}\left({ }^{n} E ; F\right), \widehat{d}^{k} P(a) \in \mathcal{P}_{H}\left({ }^{k} E ; F\right)$ and

$$
\left\|\frac{1}{k !} \widehat{d}^{k} P(a)\right\|_{H} \leq \sigma^{n}\|P\|_{H}\|a\|^{n-k},
$$

where $\widehat{d}^{k} P(a)$ is the $k$ th differential of $P$ at $a$ (see $\left.[12,19]\right)$.

If we have quasi-norms instead of norms (each $\mathcal{P}_{H}\left({ }^{n} E ; F\right)$ is a complete quasi-normed space with quasi-norm constants not depending on the underlying spaces $E$ and $F$, but possibly depending on $n$ ), we say that $\mathcal{P}_{H}$ is a global quasi-holomorphy type.

REMARK 1.2. Let $\mathcal{P}_{H}$ be a global (quasi-)holomorphy type with constant $\sigma$ and $\|\cdot\|_{K}$ be another (quasi-)norm on $\mathcal{P}_{H}$ for which there is $\gamma \geq 1$ such that

$$
\|P\|_{H} \leq\|P\|_{K} \leq \gamma^{n}\|P\|_{H}
$$

for all $n, E, F$ and $P \in \mathcal{P}_{H}\left({ }^{n} E ; F\right)$. It is clear that $\left(\mathcal{P}_{H},\|\cdot\|_{K}\right)$ is a global (quasi-)holomorphy type with constant $\sigma \gamma$. In many applications $\gamma$ will be the Euler number $e$.

Definition 1.3. An ideal of multilinear mappings is a class $\mathcal{M}$ of continuous multilinear mappings between Banach spaces such that for all $n \in \mathbb{N}$ and Banach spaces $E_{1}, \ldots, E_{n}$ and $F$, the components $\mathcal{M}\left(E_{1}, \ldots, E_{n} ; F\right):=$ $\mathcal{L}\left(E_{1}, \ldots, E_{n} ; F\right) \cap \mathcal{M}$ satisfy:

(i) $\mathcal{M}\left(E_{1}, \ldots, E_{n} ; F\right)$ is a linear subspace of $\mathcal{L}\left(E_{1}, \ldots, E_{n} ; F\right)$ which contains the $n$-linear mappings of finite type,

(ii) the ideal property: if $A \in \mathcal{M}\left(E_{1}, \ldots, E_{n} ; F\right), u_{j} \in \mathcal{L}\left(G_{j} ; E_{j}\right)$ for $j=1, \ldots, n$ and $t \in \mathcal{L}(F ; H)$, then $t \circ A \circ\left(u_{1}, \ldots, u_{n}\right)$ is in $\mathcal{M}\left(G_{1}, \ldots\right.$, $\left.G_{n} ; H\right)$.

If $\|\cdot\|_{\mathcal{M}}: \mathcal{M} \rightarrow \mathbb{R}^{+}$satisfies

$\left(\mathrm{i}^{\prime}\right)$ for each natural $n$ there is $0<p_{n} \leq 1$ such that $\|\cdot\|_{\mathcal{M}}$ restricted to $\mathcal{M}\left(E_{1}, \ldots, E_{n} ; F\right)$ is a $p_{n}$-norm for all Banach spaces $E_{1}, \ldots, E_{n}$ and $F$,

(ii') $\left\|A: \mathbb{K}^{n} \rightarrow \mathbb{K}: A\left(\lambda_{1}, \ldots, \lambda_{n}\right)=\lambda_{1} \cdots \lambda_{n}\right\|_{\mathcal{M}}=1$ for all $n$,

(iii') if $A \in \mathcal{M}\left(E_{1}, \ldots, E_{n} ; F\right), u_{j} \in \mathcal{L}\left(G_{j} ; E_{j}\right)$ for $j=1, \ldots, n$ and $t \in \mathcal{L}(F ; H)$, then $\left\|t \circ A \circ\left(u_{1}, \ldots, u_{n}\right)\right\|_{\mathcal{M}} \leq\|t\|\|A\|_{\mathcal{M}}\left\|u_{1}\right\| \cdots\left\|u_{n}\right\|$,

then $\mathcal{M}$ is called a quasi-normed (normed if all $p_{n}=1$ ) ideal of multilinear mappings. Quasi-Banach (Banach if all $p_{n}=1$ ) ideals of multilinear mappings are defined in the obvious way. For a fixed ideal $\mathcal{M}$ of multilinear mappings and $n \in \mathbb{N}$, the class $\mathcal{M}_{n}:=\bigcup_{E_{1}, \ldots, E_{n}, F} \mathcal{M}\left(E_{1}, \ldots, E_{n} ; F\right)$ is called an ideal of n-linear mappings. 
A Banach ideal $\mathcal{M}_{n}$ of $n$-linear mappings is said to be closed if its norm is the usual sup norm and each component $\mathcal{M}\left(E_{1}, \ldots, E_{n} ; F\right)$ is a closed subspace of $\mathcal{L}\left(E_{1}, \ldots, E_{n} ; F\right)$. A Banach ideal $\mathcal{M}$ of multilinear mappings is closed if each $\mathcal{M}_{n}$ is closed.

DeFinition 1.4. An ideal of homogeneous polynomials, or simply an ideal of polynomials, is a class $\mathcal{Q}$ of continuous homogeneous polynomials between Banach spaces such that for all $n \in \mathbb{N}$ and Banach spaces $E$ and $F$, the components $\mathcal{Q}\left({ }^{n} E ; F\right):=\mathcal{P}\left({ }^{n} E ; F\right) \cap \mathcal{Q}$ satisfy:

(i) $\mathcal{Q}\left({ }^{n} E ; F\right)$ is a linear subspace of $\mathcal{P}\left({ }^{n} E ; F\right)$ which contains the $n$ homogeneous polynomials of finite type,

(ii) the ideal property: if $u \in \mathcal{L}(G ; E), P \in \mathcal{Q}\left({ }^{n} E ; F\right)$ and $t \in \mathcal{L}(F ; H)$, then $t \circ P \circ u$ is in $\mathcal{Q}\left({ }^{n} G ; H\right)$.

If $\|\cdot\|_{\mathcal{Q}}: \mathcal{Q} \rightarrow \mathbb{R}^{+}$satisfies

$\left(\mathrm{i}^{\prime}\right)$ for each natural $n$ there is $0<p_{n} \leq 1$ such that $\|\cdot\|_{\mathcal{Q}}$ restricted to $\mathcal{Q}\left({ }^{n} E ; F\right)$ is a $p_{n}$-norm for all Banach spaces $E$ and $F$,

(ii') $\left\|P: \mathbb{K} \rightarrow \mathbb{K}: P(\lambda)=\lambda^{n}\right\|_{\mathcal{Q}}=1$ for all $n$,

(iii') if $u \in \mathcal{L}(G ; E), P \in \mathcal{Q}\left({ }^{n} E ; F\right)$ and $t \in \mathcal{L}(F ; H)$, then $\|t \circ P \circ u\|_{\mathcal{Q}} \leq$ $\|t\|\|P\|_{\mathcal{Q}}\|u\|^{n}$,

then $\mathcal{Q}$ is called a quasi-normed (normed if all $p_{n}=1$ ) ideal of polynomials. Quasi-Banach (Banach if all $p_{n}=1$ ) ideals of polynomials are defined in the obvious way. For a fixed ideal $\mathcal{Q}$ of polynomials and $n \in \mathbb{N}$, the class $\mathcal{Q}_{n}:=\bigcup_{E, F} \mathcal{Q}\left({ }^{n} E ; F\right)$ is called an ideal of n-homogeneous polynomials. If $\mathcal{Q}$ is an ideal of polynomials, we set $\mathcal{Q}\left({ }^{0} E ; F\right)=F$ for every $E$ and $F$. In particular, if $\left(\mathcal{Q}_{n}\right)_{n=1}^{\infty}$ is a sequence of ideals of $n$-homogeneous polynomials, when we write $\left(\mathcal{Q}_{n}\right)_{n=0}^{\infty}$ it is understood that $\mathcal{Q}_{0}\left({ }^{0} E ; F\right)=F$ for every $E$ and $F$.

Closed ideals of polynomials are defined similarly to the case of ideals of multilinear mappings.

For any ideal of multilinear mappings $\mathcal{M}$ such that each $\mathcal{M}_{n}$ is a complete $p_{n}$-normed ideal of $n$-linear mappings, defining

$$
\mathcal{P}_{\mathcal{M}}:=\{P: \check{P} \in \mathcal{M}\}, \quad\|P\|_{\mathcal{P}_{\mathcal{M}}}:=\|\check{P}\|_{\mathcal{M}},
$$

we obtain a quasi-Banach (Banach if $\mathcal{M}$ is Banach) ideal of polynomials (see [9, Proposition 2.5.2]), called the ideal of polynomials generated by $\mathcal{M}$.

REMARK 1.5. The components of either a global holomorphy type, or an ideal of multilinear mappings, or an ideal of polynomials, are always spaces of mappings between Banach spaces over the same fixed field $\mathbb{K}$.

2. Examples and counterexamples. Examples of global holomorphy types and of ideals of multilinear mappings/polynomials are easily found in 
the literature. In this section we restrict ourselves to examples which show that these two categories are incomparable.

ExAmple 2.1. For $n=1$ define $\mathcal{P}_{H}\left({ }^{n} E ; F\right):=\mathcal{L}(E ; F)$ with the sup norm for every $E$ and $F$. For $n>1$, define $\mathcal{P}_{H}\left({ }^{n} E ; F\right):=\mathcal{P}\left({ }^{n} E ; F\right)$ with the sup norm if $E=F=\ell_{1}$ and $\mathcal{P}_{H}\left({ }^{n} E ; F\right):=\{0\}$ otherwise. It is obvious that $\mathcal{P}_{H}$ is a global holomorphy type which is not an ideal of polynomials. More natural examples will appear later (cf. Example 8.3 and Remark 8.6(c)).

Example 2.2. An $n$-linear mapping $A \in \mathcal{L}\left(E_{1}, \ldots, E_{n} ; F\right)$ is said to be absolutely $(1 ; 1)$-summing, written $A \in \mathcal{L}_{\mathrm{as}(1 ; 1)}\left(E_{1}, \ldots, E_{n} ; F\right)$, if the sequence $\left(A\left(x_{j}^{1}, \ldots, x_{j}^{n}\right)\right)_{j=1}^{\infty}$ is absolutely summable in $F$ whenever $\left(x_{j}^{k}\right)_{j=1}^{\infty}$ are weakly summable in $E_{k}, k=1, \ldots, n$. The characterization by means of inequalities, which defines a natural ideal norm on $\mathcal{L}_{\text {as }(1 ; 1)}\left(E_{1}, \ldots, E_{n} ; F\right)$, can be found in [1, Theorem 3.5].

Let $E$ be an infinite-dimensional Banach space with the Orlicz property (that is, the identity operator on $E$ is absolutely $(2 ; 1)$-summing), and choose $a \in E$ and $\varphi \in E^{\prime}$ such that $\varphi(a)=1$. Putting $P(x)=\varphi(x) x$ we obtain $P \in \mathcal{P}\left({ }^{2} E ; E\right)$. We have $P \in \mathcal{P}_{\mathcal{L}_{\text {as }(1 ; 1)}}\left({ }^{2} E ; E\right)$ because $\mathcal{L}_{\text {as }(1 ; 1)}\left({ }^{n} E ; E\right)=$ $\mathcal{L}\left({ }^{n} E ; E\right)$ for every $n \geq 2[1$, Proposition 3.8]. For every $x \in E$, we have $x=2 \check{P}(a, x)-\varphi(x) a$. Since $\varphi(\cdot) a$ is a finite rank operator and the identity operator $\operatorname{id}_{E} \notin \mathcal{L}_{\text {as }(1 ; 1)}(E ; E)$ (see [10, Theorem 2.18]), it follows that $\check{P}(a, \cdot) \notin \mathcal{L}_{\text {as }(1 ; 1)}(E ; E)$. But $d P(a)=2 \check{P}(a, \cdot)$, so the ideal of polynomials generated by the ideal of all absolutely $(1 ; 1)$-summing $n$-linear mappings, $n \in \mathbb{N}$, is not a global holomorphy type.

REMARK 2.3. S. Dineen [11] introduced some refinements in the definiton of holomorphy types, such as $\alpha$-holomorphy types (see [11, Definition 4]), which are, in some sense, closer to ideals of polynomials. For instance, the type $\mathcal{P}_{H}$ of Example 2.1 is not an $\alpha$-holomorphy type.

3. Property (B) and holomorphy types. In his thesis [6], the second named author proved that, under a certain condition, the ideal of polynomials generated by an ideal of multilinear mappings is a global holomorphy type. Inspired by [6, Satz 3.3.4], in this section we prove that the same holds true with a weaker condition.

Definition 3.1. For the sake of simplicity, we shall write $\left({ }^{n} E, G ; F\right)$ instead of $(E, \stackrel{(n)}{.}, E, G ; F)$.

- An $(n+1)$-linear mapping $A \in \mathcal{L}\left({ }^{n} E, G ; F\right)$ is said to be symmetric in the first $n$ variables if $A\left(x_{1}, \ldots, x_{n}, y\right)=A\left(x_{\sigma(1)}, \ldots, x_{\sigma(n)}, y\right)$ for every permutation $\sigma$ of the set $\{1, \ldots, n\}$, any $x_{1}, \ldots, x_{n} \in E$ and $y \in G$. 
- Given $A \in \mathcal{L}\left(E_{1}, \ldots, E_{n} ; F\right)$ and $a \in E_{n}$, we define $A a \in \mathcal{L}\left(E_{1}, \ldots\right.$, $\left.E_{n-1} ; F\right)$ by $A a\left(x_{1}, \ldots, x_{n-1}\right)=A\left(x_{1}, \ldots, x_{n-1}, a\right)$.

- Let $\mathcal{J}$ be a class of continuous multilinear mappings between Banach spaces such that for all $n \in \mathbb{N}$ and Banach spaces $E_{1}, \ldots, E_{n}$ and $F$, the component $\mathcal{J}\left(E_{1}, \ldots, E_{n} ; F\right):=\mathcal{L}\left(E_{1}, \ldots, E_{n} ; F\right) \cap \mathcal{J}$ is a linear subspace of $\mathcal{L}\left(E_{1}, \ldots, E_{n} ; F\right)$ equipped with a quasi-norm denoted by $\|\cdot\|_{\mathcal{J}}$. We say that $\mathcal{J}$ has property $(\mathrm{B})$ if there is $C \geq 1$ such that for every $n \in \mathbb{N}$, any Banach spaces $E$ and $F$ and every $A \in \mathcal{J}\left({ }^{n} E, \mathbb{K} ; F\right)$ symmetric in the first $n$ variables, $A 1 \in \mathcal{J}\left({ }^{n} E ; F\right)$ and $\|A 1\|_{\mathcal{J}} \leq C\|A\|_{\mathcal{J}}$.

Given $a \in E$ and $k \in \mathbb{N}$, by $a^{k}$ we mean $a, a, \ldots, a$ where $a$ appears $k$ times.

THEOREM 3.2. If the Banach ideal $\mathcal{M}$ of multilinear mappings has property (B) with constant $C$, then the Banach ideal $\mathcal{P}_{\mathcal{M}}$ of polynomials is a global holomorphy type with constant $\sigma=2 C$.

Proof. Let $a \in E$ and $A \in \mathcal{M}\left({ }^{n+1} E ; F\right)$ be a symmetric $(n+1)$-linear mapping. Choosing $\varphi: \mathbb{K} \rightarrow E$ with $\varphi(1)=a$ and $B\left(x_{1}, \ldots, x_{n}, \lambda\right)=$ $A\left(x_{1}, \ldots, x_{n}, \varphi(\lambda)\right)$, using the ideal property and property (B) we get $A a=$ $B 1 \in \mathcal{M}\left({ }^{n} E ; F\right)$ and

$$
\|A a\|_{\mathcal{M}}=\|B 1\|_{\mathcal{M}} \leq C\|B\|_{\mathcal{M}} \leq C\|A\|_{\mathcal{M}}\|\varphi\|=C\|A\|_{\mathcal{M}}\|a\|
$$

Now, let $P \in \mathcal{P}_{\mathcal{M}}\left({ }^{n} E ; F\right), k \leq n$ and $a \in E$ be given. Then $\check{P} \in$ $\mathcal{M}\left({ }^{n} E ; F\right)$ is symmetric, therefore $\check{P}_{a} \in \mathcal{M}\left({ }^{n-1} E ; F\right)$ and

$$
\|\check{P} a\|_{\mathcal{M}} \leq C\|\check{P}\|_{\mathcal{M}}\|a\|=C\|P\|_{\mathcal{P}_{\mathcal{M}}}\|a\| \text {. }
$$

If $P a \in \mathcal{P}\left({ }^{n-1} E ; F\right)$ is defined by $P a(x)=\check{P}\left(a, x^{n-1}\right)$, it is easy to see that $(P a)^{\vee}=\check{P} a$, thus $P a \in \mathcal{P}_{\mathcal{M}}\left({ }^{n-1} E ; F\right)$ and

$$
\|P a\|_{\mathcal{P}_{\mathcal{M}}}=\left\|(P a)^{\vee}\right\|_{\mathcal{M}}=\|\check{P} a\|_{\mathcal{M}} \leq C\|P\|_{\mathcal{P}_{\mathcal{M}}}\|a\| .
$$

For $1 \leq j \leq n$, define $P a^{j} \in \mathcal{P}\left({ }^{n-j} E ; F\right)$ by $P a^{j}(x)=\check{P}\left(a^{j}, x^{n-j}\right)$. It is trivial to check that $P a^{2}=(P a) a$, so

$$
\left\|P a^{2}\right\|_{\mathcal{P}_{\mathcal{M}}}=\|(P a) a\|_{\mathcal{P}_{\mathcal{M}}} \leq C\|P a\|_{\mathcal{P}_{\mathcal{M}}}\|a\| \leq C^{2}\|P\|_{\mathcal{P}_{\mathcal{M}}}\|a\|^{2} .
$$

By iteration of this procedure, it follows that $P a^{j} \in \mathcal{P}_{\mathcal{M}}\left({ }^{n-j} E ; F\right)$ and

$$
\left\|P a^{j}\right\|_{\mathcal{P}_{\mathcal{M}}} \leq C^{j}\|P\|_{\mathcal{P}_{\mathcal{M}}}\|a\|^{j} \leq C^{n}\|P\|_{\mathcal{P}_{\mathcal{M}}}\|a\|^{j}
$$

From

$$
\widehat{d}^{k} P(a)(x)=\frac{n !}{(n-k) !} \check{P}\left(a^{n-k}, x^{k}\right)
$$

we obtain

$$
\frac{1}{k !} \widehat{d}^{k} P(a)(x)=\left(\begin{array}{l}
n \\
k
\end{array}\right) \check{P}\left(a^{n-k}, x^{k}\right)=\left(\begin{array}{l}
n \\
k
\end{array}\right) P a^{n-k}(x) .
$$


It follows that $\widehat{d}^{k} P(a) \in \mathcal{P}_{\mathcal{M}}\left({ }^{n-k} E ; F\right)$ and

$$
\left\|\frac{1}{k !} \widehat{d}^{k} P(a)\right\|_{\mathcal{P}_{\mathcal{M}}} \leq 2^{n}\left\|P a^{n-k}\right\|_{\mathcal{P}_{\mathcal{M}}} \leq(2 C)^{n}\|P\|_{\mathcal{P}_{\mathcal{M}}}\|a\|^{n-k}
$$

REMARK 3.3. Observing that the proof of Theorem 3.2 does not use the triangle inequality, we conclude that if $\mathcal{M}$ is a quasi-Banach ideal of multilinear mappings such that each $\mathcal{M}_{n}$ is a complete $p_{n}$-normed ideal of $n$-linear mappings and $\mathcal{M}$ has property (B) with constant $C$, then the quasi-Banach ideal $\mathcal{P}_{\mathcal{M}}$ of polynomials is a global quasi-holomorphy type with constant $\sigma=2 C$.

Along the paper we will provide plenty of examples of ideals of multilinear mappings having property (B). In particular, the following two sections show that the ideals of multilinear mappings which are generated by the two general methods introduced by A. Pietsch [25] have property (B).

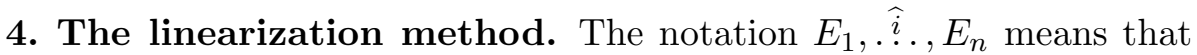
$E_{i}$ is omitted, and the same for $\left(x_{1}, \hat{i} . \stackrel{,}{.} x_{n}\right)$. For $i=1, \ldots, n$, consider the isometric isomorphism $I_{i}: \mathcal{L}\left(E_{1}, \ldots, E_{n} ; F\right) \rightarrow \mathcal{L}\left(E_{i} ; \mathcal{L}\left(E_{1},{ }_{.}{ }_{.}, E_{n} ; F\right)\right)$,

$$
I_{i}(A)\left(x_{i}\right)\left(x_{1}, \hat{i} ., x_{n}\right)=A\left(x_{1}, \ldots, x_{n}\right) .
$$

For the case $n=1$ to make sense, we set $I_{1}(A)=A$ for $A \in \mathcal{L}(E ; F)$.

Given a sequence $\left(\mathcal{I}_{n}\right)_{n=1}^{\infty}$ of Banach operator ideals, an $n$-linear mapping $A \in \mathcal{L}\left(E_{1}, \ldots, E_{n} ; F\right)$ is said to be of type $\left[\mathcal{I}_{1}, \ldots, \mathcal{I}_{n}\right]$, written $A \in$ $\left[\mathcal{I}_{1}, \ldots, \mathcal{I}_{n}\right]\left(E_{1}, \ldots, E_{n} ; F\right)$, if

$$
I_{i}(A) \in \mathcal{I}_{i}\left(E_{i} ; \mathcal{L}\left(E_{1}, . \hat{i} ., E_{n} ; F\right)\right) \quad \text { for every } i=1, \ldots, n .
$$

For $A \in\left[\mathcal{I}_{1}, \ldots, \mathcal{I}_{n}\right]\left(E_{1}, \ldots, E_{n} ; F\right)$ we define

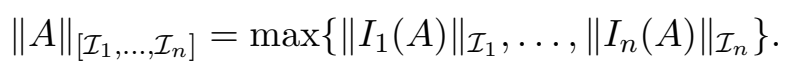

For every $n,\left[\mathcal{I}_{1}, \ldots, \mathcal{I}_{n}\right]$ is a Banach ideal of $n$-linear mappings, hence $\left(\left[\mathcal{I}_{1}, \ldots, \mathcal{I}_{n}\right]\right)_{n=1}^{\infty}$ is a Banach ideal of multilinear mappings and $\left(\mathcal{P}_{\left[\mathcal{I}_{1}, \ldots, \mathcal{I}_{n}\right]}\right)_{n=1}^{\infty}$ is a Banach ideal of polynomials; the proofs can be found in [9, Proposition 2.3.3].

THEOREM 4.1. For every sequence $\left(\mathcal{I}_{n}\right)_{n=1}^{\infty}$ of Banach operator ideals, the Banach ideal $\left(\left[\mathcal{I}_{1}, \ldots, \mathcal{I}_{n}\right]\right)_{n=1}^{\infty}$ of multilinear mappings has property $(\mathrm{B})$ with constant $C=1$. Therefore, the ideal $\left(\mathcal{P}_{\left[\mathcal{I}_{1}, \ldots, \mathcal{I}_{n}\right]}\right)_{n=0}^{\infty}$ of polynomials is a global holomorphy type with constant $\sigma=2$.

Proof. The second assertion follows from the first by Theorem 3.2. Let $A \in\left[\mathcal{I}_{1}, \ldots, \mathcal{I}_{n+1}\right]\left(E_{1}, \ldots, E_{n}, \mathbb{K} ; F\right)$. So, for $j=1, \ldots, n, I_{j}(A) \in \mathcal{I}_{j}\left(E_{j}\right.$;

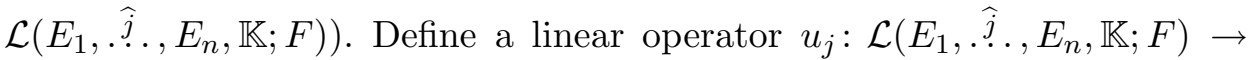


$\mathcal{L}\left(E_{1}, \stackrel{\widehat{j}}{.}, E_{n} ; F\right)$ by

$$
B \mapsto u_{j}(B)\left(x_{1}, . \stackrel{\widehat{j}}{.}, x_{n}\right)=B\left(x_{1}, \stackrel{\widehat{j}}{.}, x_{n}, 1\right) .
$$

So we have

$$
\begin{aligned}
& \left(u_{j} \circ I_{j}(A)\right)\left(x_{j}\right)\left(x_{1}, \stackrel{\widehat{j}}{.}, x_{n}\right)
\end{aligned}
$$

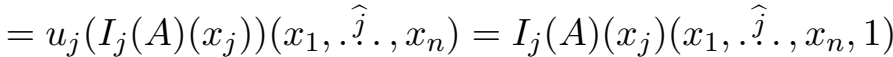

$$
\begin{aligned}
& =A\left(x_{1}, \ldots, x_{n}, 1\right)=A 1\left(x_{1}, \ldots, x_{n}\right)=I_{j}(A 1)\left(x_{j}\right)\left(x_{1}, \widehat{\jmath} . ., x_{n}\right) .
\end{aligned}
$$

We proved that $I_{j}(A 1)=u_{j} \circ I_{j}(A)$, so by the ideal property $I_{j}(A 1) \in \mathcal{I}_{j}$ for all $j=1, \ldots, n$, as $I_{j}(A) \in \mathcal{I}_{j}$. Therefore $A 1 \in\left[\mathcal{I}_{1}, \ldots, \mathcal{I}_{n}\right]\left(E_{1}, \ldots, E_{n} ; F\right)$. Moreover,

$$
\left\|I_{j}(A 1)\right\|_{\mathcal{I}_{j}}=\left\|u_{j} \circ I_{j}(A)\right\|_{\mathcal{I}_{j}} \leq\left\|u_{j}\right\|\left\|I_{j}(A)\right\|_{\mathcal{I}_{j}}=\left\|I_{j}(A)\right\|_{\mathcal{I}_{j}} .
$$

Since $I_{n+1}(A)$ is defined on $\mathbb{K}$, we have $\left\|I_{n+1}(A)\right\|_{\mathcal{I}_{n+1}}=\left\|I_{n+1}(A)\right\|=\|A\|=$ $\left\|I_{j}(A)\right\| \leq\left\|I_{j}(A)\right\|_{\mathcal{I}_{j}}, j=1, \ldots, n$. So,

$$
\begin{aligned}
\|A 1\|_{\left[\mathcal{I}_{1}, \ldots, \mathcal{I}_{n}\right]} & =\max \left\{\left\|I_{1}(A 1)\right\|_{\mathcal{I}_{1}}, \ldots,\left\|I_{n}(A 1)\right\|_{\mathcal{I}_{n}}\right\} \\
& \leq \max \left\{\left\|I_{1}(A)\right\|_{\mathcal{I}_{1}}, \ldots,\left\|I_{n}(A)\right\|_{\mathcal{I}_{n}}\right\} \\
& =\max \left\{\left\|I_{1}(A)\right\|_{\mathcal{I}_{1}}, \ldots,\left\|I_{n+1}(A)\right\|_{\mathcal{I}_{n+1}}\right\}=\|A\|_{\left[\mathcal{I}_{1}, \ldots, \mathcal{I}_{n}, \mathcal{I}_{n+1}\right]},
\end{aligned}
$$

completing the proof.

5. The factorization method. Given a sequence $\left(\mathcal{I}_{n}\right)_{n=1}^{\infty}$ of Banach operator ideals, we say that an $n$-linear mapping $A \in \mathcal{L}\left(E_{1}, \ldots, E_{n} ; F\right)$ is of type $\mathcal{L}\left(\mathcal{I}_{1}, \ldots, \mathcal{I}_{n}\right)$ if there are Banach spaces $G_{1}, \ldots, G_{n}$, linear operators $u_{j} \in \mathcal{I}_{j}\left(E_{j} ; G_{j}\right), j=1, \ldots, n$, and a continuous $n$-linear mapping $B \in$ $\mathcal{L}\left(G_{1}, \ldots, G_{n} ; F\right)$ such that $A=B \circ\left(u_{1}, \ldots, u_{n}\right)$. In this case we write $A \in \mathcal{L}\left(\mathcal{I}_{1}, \ldots, \mathcal{I}_{n}\right)\left(E_{1}, \ldots, E_{n} ; F\right)$, and define

$$
\|A\|_{\mathcal{L}\left(\mathcal{I}_{1}, \ldots, \mathcal{I}_{n}\right)}=\inf \|B\|\left\|u_{1}\right\|_{\mathcal{I}_{1}} \cdots\left\|u_{n}\right\|_{\mathcal{I}_{n}}
$$

where the infimum is taken over all possible factorizations $A=B \circ\left(u_{1}, \ldots, u_{n}\right)$ with $u_{j} \in \mathcal{I}_{j}$.

For every $n, \mathcal{L}\left(\mathcal{I}_{1}, \ldots, \mathcal{I}_{n}\right)$ is a complete $1 / n$-normed ideal of $n$-linear mappings, hence $\left(\mathcal{L}\left(\mathcal{I}_{1}, \ldots, \mathcal{I}_{n}\right)\right)_{n=1}^{\infty}$ is a quasi-Banach ideal of multilinear mappings and $\left(\mathcal{P}_{\mathcal{L}\left(\mathcal{I}_{1}, \ldots, \mathcal{I}_{n}\right)}\right)_{n=1}^{\infty}$ is a quasi-Banach ideal of polynomials; the proofs can be found in [9, Proposition 2.3.3].

It is noteworthy that if $\mathcal{I}_{1}, \ldots, \mathcal{I}_{n}$ are closed operator ideals, then $\|\cdot\|_{\mathcal{L}\left(\mathcal{I}_{1}, \ldots, \mathcal{I}_{n}\right)}$ is a norm (this fact was first proved in [6, Satz 2.5.7]) and the components $\mathcal{L}\left(\mathcal{I}_{1}, \ldots, \mathcal{I}_{n}\right)\left(E_{1}, \ldots, E_{n} ; F\right)$ are closed (see [7, Proposition 3.5]). Actually, in this case, from [14, Lemma 1.2] (or [15, Lemma 6]) it is easy to see that $\|A\|=\|A\|_{\mathcal{L}\left(\mathcal{I}_{1}, \ldots, \mathcal{I}_{n}\right)}$ for all $A \in \mathcal{L}\left(\mathcal{I}_{1}, \ldots, \mathcal{I}_{n}\right)\left(E_{1}, \ldots, E_{n} ; F\right)$. 
Theorem 5.1. For every sequence $\left(\mathcal{I}_{n}\right)_{n=1}^{\infty}$ of Banach operator ideals, the quasi-Banach ideal $\left(\mathcal{L}\left(\mathcal{I}_{1}, \ldots, \mathcal{I}_{n}\right)\right)_{n=1}^{\infty}$ of multilinear mappings has property (B) with constant $C=1$. Therefore, the ideal $\left(\mathcal{P}_{\mathcal{L}\left(\mathcal{I}_{1}, \ldots, \mathcal{I}_{n}\right)}\right)_{n=0}^{\infty}$ of polynomials is a global quasi-holomorphy type with constant $\sigma=2$.

Proof. The second assertion follows from the first by Remark 3.3. Given an $(n+1)$-linear mapping $A \in \mathcal{L}\left(\mathcal{I}_{1}, \ldots, \mathcal{I}_{n+1}\right)\left(E_{1}, \ldots, E_{n}, \mathbb{K} ; F\right)$, there exist Banach spaces $G_{1}, \ldots, G_{n}, G$, linear operators $u \in \mathcal{I}_{n+1}(\mathbb{K} ; G), u_{j} \in$ $\mathcal{I}_{j}\left(E_{j} ; G_{j}\right), j=1, \ldots, n$, and $B \in \mathcal{L}\left(G_{1}, \ldots, G_{n}, G ; F\right)$ such that $A=$ $B \circ\left(u_{1}, \ldots, u_{n}, u\right)$. Defining

$$
D: G_{1} \times \cdots \times G_{n} \rightarrow F, \quad D\left(y_{1}, \ldots, y_{n}\right)=B\left(y_{1}, \ldots, y_{n}, u(1)\right),
$$

we get

$$
\begin{aligned}
A 1\left(x_{1}, \ldots, x_{n}\right) & =A\left(x_{1}, \ldots, x_{n}, 1\right)=B\left(u_{1}\left(x_{1}\right), \ldots, u_{n}\left(x_{n}\right), u(1)\right) \\
& =D\left(u_{1}\left(x_{1}\right), \ldots, u_{n}\left(x_{n}\right)\right)=\left(D \circ\left(u_{1}, \ldots, u_{n}\right)\right)\left(x_{1}, \ldots, x_{n}\right),
\end{aligned}
$$

showing that $A 1=D \circ\left(u_{1}, \ldots, u_{n}\right)$. Since each $u_{j}$ belongs to $\mathcal{I}_{j}$ it follows that $A 1 \in \mathcal{L}\left(\mathcal{I}_{1}, \ldots, \mathcal{I}_{n}\right)\left(E_{1}, \ldots, E_{n} ; F\right)$. Moreover, from $\|D\| \leq\|B\|\|u\|$ and $\|u\| \leq\|u\|_{\mathcal{I}_{n+1}}$ (see $[10$, p. 131]), we have

$$
\|A 1\|_{\mathcal{L}\left(\mathcal{I}_{1}, \ldots, \mathcal{I}_{n}\right)} \leq\|D\|\left\|u_{1}\right\|_{\mathcal{I}_{1}} \cdots\left\|u_{n}\right\|_{\mathcal{I}_{n}} \leq\|B\|\|u\|_{\mathcal{I}_{n+1}}\left\|u_{1}\right\|_{\mathcal{I}_{1}} \cdots\left\|u_{n}\right\|_{\mathcal{I}_{n}} .
$$

So, $\|A 1\|_{\mathcal{L}\left(\mathcal{I}_{1}, \ldots, \mathcal{I}_{n}\right)} \leq\|B\|\left\|u_{1}\right\|_{\mathcal{I}_{1}} \cdots\left\|u_{n}\right\|_{\mathcal{I}_{n}}\|u\|_{\mathcal{I}_{n+1}}$ for every representation $A=B \circ\left(u_{1}, \ldots, u_{n}, u\right)$ with $u_{j} \in \mathcal{I}_{j}, j=1, \ldots, n$ and $u \in \mathcal{I}_{n+1}$. We finish the proof by taking the infimum over all such representations to obtain $\|A 1\|_{\mathcal{L}\left(\mathcal{I}_{1}, \ldots, \mathcal{I}_{n}\right)} \leq\|A\|_{\mathcal{L}\left(\mathcal{I}_{1}, \ldots, \mathcal{I}_{n+1}\right)}$.

In the special case where $\mathcal{I}_{n}=\mathcal{I}$ for every $n \in \mathbb{N}$, we shall write $\mathcal{L}(\mathcal{I})$ and $\mathcal{P}_{\mathcal{L}(\mathcal{I})}$ instead of $\mathcal{L}(\mathcal{I}, \ldots, \mathcal{I})$ and $\mathcal{P}_{\mathcal{L}(\mathcal{I}, \ldots, \mathcal{I})}$. Until now, in $\mathcal{P}_{\mathcal{L}(\mathcal{I})}$ we have considered the $1 / n$-norm $P \in \mathcal{P}_{\mathcal{L}(\mathcal{I})}\left({ }^{n} E ; F\right) \mapsto\|P\|_{\mathcal{L}(\mathcal{I})}=\|\check{P}\|_{\mathcal{L}(\mathcal{I})}$. It is well known that $P \in \mathcal{P}_{\mathcal{L}(\mathcal{I})}\left({ }^{n} E ; F\right) \Leftrightarrow$ there exists $A \in \mathcal{L}(\mathcal{I})\left({ }^{n} E ; F\right)$ such that $\widehat{A}=P \Leftrightarrow$ there are a Banach space $G$, a linear operator $u \in \mathcal{I}(E ; G)$ and a polynomial $Q \in \mathcal{P}\left({ }^{n} G ; F\right)$ such that $P=Q \circ u$ (see [3, Proposition 4.3]). So, for $P \in \mathcal{P}_{\mathcal{L}(\mathcal{I})}\left({ }^{n} E ; F\right)$ we can consider

$$
\begin{aligned}
\|P\|_{\mathcal{L}(\mathcal{I}), 1} & :=\inf \left\{\|A\|_{\mathcal{L}(\mathcal{I})}: \widehat{A}=P\right\}, \\
\|P\|_{\mathcal{L}(\mathcal{I}), 2} & :=\inf \left\{\|Q\|\|u\|_{\mathcal{I}}^{n}: P=Q \circ u, u \in \mathcal{I}\right\},
\end{aligned}
$$

which define two other complete $1 / n$-norms on $\mathcal{P}_{\mathcal{L}(\mathcal{I})}\left({ }^{n} E ; F\right)$ (see $[8$, p. 50] and [9, Proposition 2.5.5]).

Theorem 5.2. For every Banach operator ideal $\mathcal{I}$, the ideal $\mathcal{P}_{\mathcal{L}(\mathcal{I})}$ of polynomials is a global quasi-holomorphy type with either $\|\cdot\|_{\mathcal{L}(\mathcal{I})},\|\cdot\|_{\mathcal{L}(\mathcal{I}), 1}$ or $\|\cdot\|_{\mathcal{L}(\mathcal{I}), 2}$.

Proof. From Theorem 5.1 we know that $\left(\mathcal{P}_{\mathcal{L}(\mathcal{I})},\|\cdot\|_{\mathcal{L}(\mathcal{I})}\right)$ is a global quasi-holomorphy type with constant $\sigma=2$. So, for every $n$, any $k \leq n$, 
$a \in E$ and $P \in \mathcal{P}_{\mathcal{L}(\mathcal{I})}\left({ }^{n} E ; F\right)$, we have $\widehat{d}^{k} P(a) \in \mathcal{P}_{\mathcal{L}(\mathcal{I})}\left({ }^{k} E ; F\right)$ and

$$
\left\|\frac{1}{k !} \widehat{d}^{k} P(a)\right\|_{\mathcal{L}(\mathcal{I})} \leq 2^{n}\|P\|_{\mathcal{L}(\mathcal{I})}\|a\|^{n-k} .
$$

Let $n \in \mathbb{N}, k \leq n, a \in E$ and $P \in \mathcal{P}_{\mathcal{L}(\mathcal{I})}\left({ }^{n} E ; F\right)$. We already know that $\widehat{d}^{k} P(a) \in \mathcal{P}_{\mathcal{L}(\mathcal{I})}\left({ }^{k} E ; F\right)$. We conclude that $\left(\mathcal{P}_{\mathcal{L}(\mathcal{I})},\|\cdot\|_{\mathcal{L}(\mathcal{I}), 1}\right)$ and $\left(\mathcal{P}_{\mathcal{L}(\mathcal{I})}\right.$, $\left.\|\cdot\|_{\mathcal{L}(\mathcal{I}), 2}\right)$ are global quasi-holomorphy types with constant $\sigma=2 e$ by observing that

$$
\begin{aligned}
\left\|\frac{1}{k !} \widehat{d}^{k} P(a)\right\|_{\mathcal{L}(\mathcal{I}), 2} & \leq\left\|\frac{1}{k !} \widehat{d}^{k} P(a)\right\|_{\mathcal{L}(\mathcal{I}), 1} \leq\left\|\frac{1}{k !} \widehat{d}^{k} P(a)\right\|_{\mathcal{L}(\mathcal{I})} \\
& \leq 2^{n}\|P\|_{\mathcal{L}(\mathcal{I})}\|a\|^{n-k} \leq(2 e)^{n}\|P\|_{\mathcal{L}(\mathcal{I}), 2}\|a\|^{n-k} \\
& \leq(2 e)^{n}\|P\|_{\mathcal{L}(\mathcal{I}), 1}\|a\|^{n-k}
\end{aligned}
$$

where the first and the last inequalities follow from [8, Lemma 3.1], the second is obvious, the third follows from $(*)$, and the fourth from $[9$, Proposition 2.5.5].

EXAMPLE 5.3. Given a sequence $\left(p_{n}\right)_{n=1}^{\infty}$ of numbers with each $p_{n} \geq 1$, we denote by $\mathcal{L}_{d ; p_{1}, \ldots, p_{n}}$ the class of all $\left(p_{1}, \ldots, p_{n}\right)$-dominated $n$-linear mappings (see $[3,4,9])$ endowed with the $\left(p_{1}, \ldots, p_{n}\right)$-dominated quasi-norm. Since $\mathcal{L}\left(\Pi_{p_{1}}, \ldots, \Pi_{p_{n}}\right)=\mathcal{L}_{d ; p_{1}, \ldots, p_{n}}$ isometrically, where $\Pi_{p}$ is the ideal of all absolutely $p$-summing linear operators, from Theorem 5.1 we see that $\left(\mathcal{L}_{d ; p_{1}, \ldots, p_{n}}\right)_{n=1}^{\infty}$ has property (B) with constant $C=1$. Therefore, given $p \geq 1$, the class $\mathcal{P}_{d, p}$ of all $p$-dominated homogeneous polynomials is a global quasi-holomorphy type, with respect to the $p$-dominated quasi-norm, with constant $\sigma=2$.

\section{Sufficient conditions for holomorphy type to imply prop-} erty (B). Let $\mathcal{M}$ be a Banach ideal of multilinear mappings. Information about polynomials in $\mathcal{P}_{\mathcal{M}}$ implies information about symmetric multilinear mappings in $\mathcal{M}$. But there are plenty of non-symmetric multilinear mappings in $\mathcal{M}$, so a full converse of Theorem 3.2 is not to be expected. Even so, we are going to see that a partial converse holds true, that is, for certain ideals, having property (B) is equivalent to generating a holomorphy type. A little preparation is needed.

Given $A \in \mathcal{L}\left({ }^{n} E ; F\right)$, we denote by $A_{S}$ the symmetrization of $A$. An ideal of multilinear mappings $\mathcal{M}$ is said to be

- symmetric if $A_{S} \in \mathcal{M}\left({ }^{n} E ; F\right)$ whenever $A \in \mathcal{M}\left({ }^{n} E ; F\right)$ (cf. [13]);

- closed for scalar multiplication (csm, for short) if $\mathcal{M}$ satisfies at least one of the following conditions (cf. [4]): 
(i) if $A \in \mathcal{M}\left({ }^{n} E ; F\right), \varphi \in E^{\prime}$ and $\varphi A \in \mathcal{L}\left({ }^{n+1} E ; F\right)$ is given by

$$
\varphi A\left(x_{1}, \ldots, x_{n+1}\right)=\varphi\left(x_{1}\right) A\left(x_{2}, \ldots, x_{n+1}\right),
$$

then $\varphi A \in \mathcal{M}\left({ }^{n+1} E ; F\right)$;

(ii) if $A \in \mathcal{M}\left({ }^{n} E ; F\right), \varphi \in E^{\prime}$ and $A \varphi \in \mathcal{L}\left({ }^{n+1} E ; F\right)$ is given by

$$
A \varphi\left(x_{1}, \ldots, x_{n+1}\right)=\varphi\left(x_{n+1}\right) A\left(x_{1}, \ldots, x_{n}\right),
$$

then $A \varphi \in \mathcal{M}\left({ }^{n+1} E ; F\right)$.

TheOREM 6.1. Let $\mathcal{M}$ be a csm symmetric closed ideal of multilinear mappings. The following assertions are equivalent.

(a) For all $n, E$ and $F, A 1 \in \mathcal{M}\left({ }^{n} E ; F\right)$ for every $A \in \mathcal{M}\left({ }^{n} E, \mathbb{K} ; F\right)$ symmetric in the first $n$ variables.

(b) $\mathcal{M}$ has property (B) (with best constant $C=1$ ).

(c) $\mathcal{P}_{\mathcal{M}}$ is a global holomorphy type (with best constant $\sigma$ not greater than 2).

Proof. (a) $\Rightarrow(\mathrm{b})$. Obvious because $\|A 1\|=\|A\|$.

(b) $\Rightarrow$ (c). This is a particular case of Theorem 3.2.

(c) $\Rightarrow$ (a). Let $A \in \mathcal{M}\left({ }^{n} E, \mathbb{K} ; F\right)$ be symmetric in the first $n$ variables. Choose $\varphi \in E^{\prime}$ and $a \in E$ such that $\varphi(a)=1$ and define $B:=A \circ\left(\operatorname{id}_{E}, \stackrel{(n)}{\text {. }}\right.$, $\left.\operatorname{id}_{E}, \varphi\right)$. Then $B \in \mathcal{M}\left({ }^{n+1} E ; F\right)$ by the ideal property. Since $\mathcal{M}$ is symmetric, $B_{S} \in \mathcal{M}\left({ }^{n+1} E ; F\right)$, hence $\widehat{B}=\widehat{B_{S}} \in \mathcal{P}_{\mathcal{M}}\left({ }^{n+1} E ; F\right)$. As $\mathcal{P}_{\mathcal{M}}$ is a global holomorphy type by assumption, $\widehat{d}^{k} \widehat{B}(a) \in \mathcal{P}_{\mathcal{M}}\left({ }^{k} E ; F\right)$ for $k=1, \ldots, n$.

For $k=0,1, \ldots, n-1$, define $P_{k} \in \mathcal{P}\left({ }^{k+1} E ; F\right)$ by

$$
P_{k}(x)=\varphi(x) A 1\left(a^{n-k}, x^{k}\right) .
$$

Let us prove, by induction on $k$, that $P_{k} \in \mathcal{P}_{\mathcal{M}}\left({ }^{k+1} E ; F\right)$ for $k=0,1, \ldots, n-1$.

For $k=0$ this is obvious.

Now we assume that $P_{k} \in \mathcal{P}_{\mathcal{M}}\left({ }^{k+1} E ; F\right)$ and prove $P_{k+1} \in \mathcal{P}_{\mathcal{M}}\left({ }^{k+2} E ; F\right)$. For every $x \in E$ and $k=0,1, \ldots, n-2$, we have

$$
\begin{aligned}
& \frac{(n-k) !}{(n+1) !} \widehat{d}^{k+1} \widehat{B}(a)(x)=B_{S}\left(a^{n-k}, x^{k+1}\right) \\
& =\frac{1}{n+1}\left[(n-k) \varphi(a) A 1\left(a^{n-k-1}, x^{k+1}\right)+(k+1) \varphi(x) A 1\left(a^{n-k}, x^{k}\right)\right] \\
& =\frac{1}{n+1}\left[(n-k) A 1\left(a^{n-k-1}, x^{k+1}\right)+(k+1) P_{k}(x)\right] .
\end{aligned}
$$

But $\widehat{d}^{k+1} \widehat{B}(a)$ and $P_{k}$ belong to $\mathcal{P}_{\mathcal{M}}\left({ }^{k+1} E ; F\right)$, so the $(k+1)$-homogeneous polynomial $Q(x):=A 1\left(a^{n-k-1}, x^{k+1}\right)$ belongs to $\mathcal{P}_{\mathcal{M}}\left({ }^{k+1} E ; F\right)$ as well. So $\check{Q} \in \mathcal{M}\left({ }^{k+1} E ; F\right)$. Since $A 1$ is symmetric (because $A$ is symmetric in the first $n$ variables $)$, it follows that $\check{Q}\left(x_{1}, \ldots, x_{k+1}\right)=A 1\left(a^{n-k-1}, x_{1}, \ldots, x_{k+1}\right)$. 
Suppose that $\mathcal{M}$ satisfies condition (i) of the definition of csm ideals. Using this condition for $\varphi$ and $\check{Q}$ we find that the $(k+2)$-linear mapping $C_{k+1}\left(x_{1}, \ldots, x_{k+2}\right):=\varphi\left(x_{1}\right) \check{Q}\left(x_{2}, \ldots, x_{k+2}\right)$ belongs to $\mathcal{M}\left({ }^{k+2} E ; F\right)$. Since $\mathcal{M}$ is symmetric, $P_{k+1}=\widehat{C_{k+1}}=\left(\widehat{C_{k+1}}\right)_{S} \in \mathcal{P}_{\mathcal{M}}\left({ }^{k+2} E ; F\right)$. If $\mathcal{M}$ satisfies condition (ii) of the definition of csm ideals, then a suitable modification of the definition of $C_{k+1}$ completes the proof by induction.

Setting $k=n-1$ we see that $P_{n-1} \in \mathcal{P}_{\mathcal{M}}\left({ }^{n} E ; F\right)$. For every $x \in E$,

$$
\begin{aligned}
\frac{1}{n !} \widehat{d}^{n} \widehat{B}(a)(x) & =(n+1) B_{S}\left(a, x^{n}\right)=\varphi(a) A 1\left(x^{n}\right)+n \varphi(x) A 1\left(a, x^{n-1}\right) \\
& =\widehat{A 1}(x)+n P_{n-1}(x) .
\end{aligned}
$$

But $\widehat{d^{n}} \widehat{B}(a)$ and $P_{n-1}$ belong to $\mathcal{P}_{\mathcal{M}}\left({ }^{n} E ; F\right)$, so $\widehat{A 1} \in \mathcal{P}_{\mathcal{M}}\left({ }^{n} E ; F\right)$ as well. Since $A 1$ is symmetric, it follows that $A 1=(\widehat{A 1})^{\vee} \in \mathcal{M}\left({ }^{n} E ; F\right)$.

REMARK 6.2 (On the definiton of property (B)). We could have used the following variant of property $(\mathrm{B})$ :

- An $(n+1)$-linear mapping $A \in \mathcal{L}(G, E, \stackrel{(n)}{.}, E ; F)$ is said to be symmetric from the second variable on if

$$
A\left(y, x_{1}, \ldots, x_{n}\right)=A\left(y, x_{\sigma(1)}, \ldots, x_{\sigma(n)}\right)
$$

for every permutation $\sigma$ of the set $\{1, \ldots, n\}$, any $x_{1}, \ldots, x_{n} \in E$ and $y \in G$.

- Given $A \in \mathcal{L}\left(E_{1}, \ldots, E_{n} ; F\right)$ and $a \in E_{1}$, we define $a A \in \mathcal{L}\left(E_{2}, \ldots\right.$, $\left.E_{n} ; F\right)$ by $a A\left(x_{2}, \ldots, x_{n}\right)=A\left(a, x_{2}, \ldots, x_{n}\right)$.

- Let $\mathcal{J}$ be a class of multilinear mappings such that each component $\mathcal{J}\left(E_{1}, \ldots, E_{n}, F\right)$ is endowed with a quasi-norm $\|\cdot\|_{\mathcal{J}}$. We say that $\mathcal{J}$ has property $\left(\mathrm{B}^{\prime}\right)$ if there is $C \geq 1$ such that for every $n \in \mathbb{N}$ and every $A \in \mathcal{J}(\mathbb{K}, E, \stackrel{(n)}{.}, E ; F)$ symmetric from the second variable on, $1 A \in \mathcal{J}\left({ }^{n} E ; F\right)$ and $\|1 A\|_{\mathcal{J}} \leq C\|A\|_{\mathcal{J}}$.

A simple adaptation of the proof of Theorem 3.2 shows that a Banach ideal of multilinear mappings having property $\left(\mathrm{B}^{\prime}\right)$ also generates a holomorphy type. It is not difficult to see that even for Banach ideals of multilinear mappings, properties $(\mathrm{B})$ and $\left(\mathrm{B}^{\prime}\right)$ are not equivalent. Nevertheless, to each ideal $\mathcal{M}$ with property $\left(\mathrm{B}^{\prime}\right)$ we can associate an ideal $\mathcal{M}^{\prime}$ with property $(\mathrm{B})$ by defining $\mathcal{M}^{\prime}\left(E_{1}, \ldots, E_{n} ; F\right):=\mathcal{M}\left(E_{n}, \ldots, E_{1} ; F\right)$.

The notion of strongly symmetric quasi-normed ideals of multilinear mappings was introduced by K. Floret and D. García [13]. It is easy to see that, for strongly symmetric ideals, properties $(\mathrm{B})$ and $\left(\mathrm{B}^{\prime}\right)$ are equivalent.

7. Weak property (B). In the proof of Theorem 6.1, we used the trivial fact that if $\mathcal{M}$ is a closed ideal of multilinear mappings and $A 1 \in \mathcal{M}$ 
whenever $A \in \mathcal{M}$, then $\mathcal{M}$ has property (B). It is natural to ask if this weak version of property $(\mathrm{B})$, namely:

$A \in \mathcal{M}\left({ }^{n} E, \mathbb{K} ; F\right)$ symmetric in the first $n$ variables $\Rightarrow A 1 \in \mathcal{M}\left({ }^{n} E ; F\right)$, implies property (B) in general. The ideal we construct in this section proves that the answer is negative, that is: in general, the norming condition $\|A 1\|_{\mathcal{M}} \leq C\|A\|_{\mathcal{M}}$ does not follow from the condition $A \in \mathcal{M} \Rightarrow A 1 \in \mathcal{M}$.

Let $\mathcal{N}$ denote the operator ideal of nuclear operators on Hilbert spaces. According to [24, Section 15.2] we can restrict ourselves to operators from a Hilbert space $H$ into itself. Given $u \in \mathcal{N}(H ; H)$, from [10, Theorem 5.30(b)] we know that $\|u\|_{\mathcal{N}}=\sum_{j=1}^{\infty} s_{j}(u)$, where $s_{j}(u)$ is the $j$ th approximation (or singular) number of $u$.

Definition 7.1. For an operator $u$ in $\mathcal{N}$ and $n \geq 1$, we put

$$
\nu_{n}(u):=s_{1}(u)+\frac{1}{(n-1) !} \sum_{j=2}^{\infty} s_{j}(u) \text {. }
$$

Proposition 7.2. Let $n \geq 1$.

(a) $\nu_{n}$ is an ideal norm on $\mathcal{N}$ equivalent to the nuclear norm $\|\cdot\|_{\mathcal{N}}=\nu_{1}$.

(b) $\nu_{n+1}(u) \leq \nu_{n}(u) \leq n \nu_{n+1}(u)$ for all $u$ in $\mathcal{N}$ and the constant $n$ is optimal in the second inequality, that is, $\sup _{0 \neq u \in \mathcal{N}} \nu_{n}(u) / \nu_{n+1}(u)$ $=n$.

Proof. (a) We only prove the triangle inequality. For any compact linear operators $u, v: H \rightarrow H$ and every $n \geq 1$, from [27, Proposition III.G.11] we know that $\sum_{j=1}^{n} s_{j}(u+v) \leq \sum_{j=1}^{n}\left(s_{j}(u)+s_{j}(v)\right)$. So, if $u, v \in \mathcal{N}(H ; H)$, then

$$
\begin{aligned}
\nu_{n}(u+v) & =s_{1}(u+v)+\frac{1}{(n-1) !} \sum_{j=2}^{\infty} s_{j}(u+v) \\
& \leq s_{1}(u)+s_{1}(v)+\frac{1}{(n-1) !} \sum_{j=2}^{\infty}\left(s_{j}(u)+s_{j}(v)\right)=\nu_{n}(u)+\nu_{n}(v) .
\end{aligned}
$$

(b) We have

$$
\begin{aligned}
\nu_{n+1}(u) & =s_{1}(u)+\frac{1}{n !} \sum_{j=2}^{\infty} s_{j}(u) \leq s_{1}(u)+\frac{1}{(n-1) !} \sum_{j=2}^{\infty} s_{j}(u) \\
& =\nu_{n}(u)=n\left(\frac{s_{1}(u)}{n}+\frac{1}{n !} \sum_{j=2}^{\infty} s_{j}(u)\right) \leq n \nu_{n+1}(u) .
\end{aligned}
$$

To see the optimality of the constant $n$, let $\left(e_{j}\right)$ be the standard unit vectors of sequence spaces and define, for each $k \in \mathbb{N}, u_{k}:=\sum_{j=1}^{k} e_{j}^{*} \otimes e_{j} \in \mathcal{N}\left(\ell_{2} ; \ell_{2}\right)$. So, 


$$
\frac{\nu_{n}\left(u_{k}\right)}{\nu_{n+1}\left(u_{k}\right)}=\frac{1+\frac{1}{(n-1) !}(k-1)}{1+\frac{1}{n !}(k-1)}=\frac{n !+n(k-1)}{n !+(k-1)} \rightarrow n \quad \text { as } k \rightarrow \infty .
$$

We denote by $\mathcal{U}$ the maximal extension of $\mathcal{N}$ to the class of all Banach spaces. By [24, Theorem 15.6.3], an operator $u: E \rightarrow F$ between Banach spaces belongs to $\mathcal{U}$ if and only if for all Hilbert spaces $H_{1}, H_{2}$ and all operators $v \in \mathcal{L}\left(H_{1} ; E\right), t \in \mathcal{L}\left(F ; H_{2}\right)$, the composition $t \circ u \circ v$ belongs to $\mathcal{N}$.

Following [24, Section 7.2], it can be easily seen that, for each $n$, the norm $\nu_{n}$ on $\mathcal{N}$ can be extended to a Banach ideal norm $\nu_{n}$ on $\mathcal{U}$ defined by $\nu_{n}(u):=\sup \left\{\nu_{n}(t \circ u \circ v):\|t\|,\|v\| \leq 1\right\}$. Proposition 7.2 implies

PROPOSITION 7.3. $\nu_{n}(u) \leq n \nu_{n+1}(u)$ for all $n$ and $u \in \mathcal{U}$, where the constant $n$ is optimal. Therefore $\sup \left\{\nu_{n}(u) / \nu_{n+1}(u): 0 \neq u \in \mathcal{U}, n \in \mathbb{N}\right\}=\infty$.

Given an operator ideal $\mathcal{I}$ and $n \in \mathbb{N}$, for convenience we shall write $\left[{ }^{n} \mathcal{I}\right]$ instead of $[\mathcal{I}, .(n), \mathcal{I}]$.

THEOREM 7.4. Let $\left(\mathcal{I}_{n}\right)_{n=1}^{\infty}$ be a sequence of Banach operator ideals such that, for every $n \in \mathbb{N}, \mathcal{I}_{n+1} \subseteq \mathcal{I}_{n}$ and $\|u\|_{\mathcal{I}_{n}} \leq C_{n}\|u\|_{\mathcal{I}_{n+1}}$ for all $u \in \mathcal{I}_{n+1}$, where $C_{n}$ is the optimal constant. Then, for all $A \in\left[{ }^{n+1} \mathcal{I}_{n+1}\right]\left(E_{1}, \ldots, E_{n}\right.$, $\mathbb{K} ; F)$ we have $A 1 \in\left[{ }^{n} \mathcal{I}_{n}\right]\left(E_{1}, \ldots, E_{n} ; F\right)$ and $\|A 1\|_{\left[{ }^{n} \mathcal{I}_{n}\right]} \leq C_{n}\|A\|_{\left[{ }^{n+1} \mathcal{I}_{n+1}\right]}$, where $C_{n}$ is the optimal constant.

Proof. Let $A \in\left[{ }^{n+1} \mathcal{I}_{n+1}\right]\left(E_{1}, \ldots, E_{n}, \mathbb{K} ; F\right)$. For $j=1, \ldots, n$, we have $I_{j}(A 1) \in \mathcal{L}\left(E_{j} ; \mathcal{L}\left(E_{1}, .{ }_{j} ., E_{n} ; F\right)\right)$. The mapping

$$
J: \mathcal{L}\left(G_{1}, \ldots, G_{n}, \mathbb{K} ; F\right) \rightarrow \mathcal{L}\left(G_{1}, \ldots, G_{n} ; F\right), \quad B \mapsto J(B):=B 1,
$$

is an isometric isomorphism. Since $I_{j}(A) \in \mathcal{I}_{n+1}\left(E_{j} ; \mathcal{L}\left(E_{1},{ }^{\hat{j}} ., E_{n}, \mathbb{K} ; F\right)\right)$ for $j=1, \ldots, n$, it follows that

$I_{j}(A 1)=J \circ I_{j}(A) \in \mathcal{I}_{n+1}\left(E_{j} ; \mathcal{L}\left(E_{1},{ }^{\hat{j}} ., E_{n} ; F\right)\right) \subseteq \mathcal{I}_{n}\left(E_{j} ; \mathcal{L}\left(E_{1},{ }^{\hat{j}} ., E_{n} ; F\right)\right)$, and $\left\|I_{j}(A 1)\right\|_{\mathcal{I}_{n}}=\left\|J \circ I_{j}(A)\right\|_{\mathcal{I}_{n}}=\left\|I_{j}(A)\right\|_{\mathcal{I}_{n}} \leq C_{n}\left\|I_{j}(A)\right\|_{\mathcal{I}_{n+1}}$. Hence $A 1 \in\left[{ }^{n} \mathcal{I}_{n}\right]\left(E_{1}, \ldots, E_{n} ; F\right)$ and $\|A 1\|_{\left[{ }^{n} \mathcal{I}_{n}\right]} \leq C_{n}\|A\|_{\left[{ }^{n+1} \mathcal{I}_{n+1}\right]}$.

Given $0<\varepsilon<C_{n}$, choose $u \in \mathcal{I}_{n+1}(E ; F)$ such that $\|u\|_{\mathcal{I}_{n}} /\|u\|_{\mathcal{I}_{n+1}} \geq$ $C_{n}-\varepsilon$. Defining $A \in \mathcal{L}(E, \mathbb{K}, . \stackrel{(n)}{.}, \mathbb{K} ; F)$ by $A\left(x, \lambda_{1}, \ldots, \lambda_{n}\right)=\lambda_{1} \cdots \lambda_{n} u(x)$ we find that $A \in\left[{ }^{n+1} \mathcal{I}_{n+1}\right]\left(E, \mathbb{K}, .{ }^{(n)}, \mathbb{K} ; F\right)$ and

$$
\begin{aligned}
\|A\|_{\left[{ }^{n+1} \mathcal{I}_{n+1}\right]} & =\max \left\{\left\|I_{1}(A)\right\|_{\mathcal{I}_{n+1}},\left\|I_{j}(A)\right\|_{\mathcal{I}_{n+1}}: 2 \leq j \leq n+1\right\} \\
& =\max \left\{\|u\|_{\mathcal{I}_{n+1}},\|u\|\right\}=\|u\|_{\mathcal{I}_{n+1}} .
\end{aligned}
$$

In the same way we obtain $\|A 1\|_{\left[{ }^{n} \mathcal{I}_{n}\right]}=\|u\|_{\mathcal{I}_{n}}$ and therefore

$$
\frac{\|A 1\|_{\left[{ }^{n} \mathcal{I}_{n}\right]}}{\|A\|_{\left[{ }^{n+1} \mathcal{I}_{n+1}\right]}}=\frac{\|u\|_{\mathcal{I}_{n}}}{\|u\|_{\mathcal{I}_{n+1}}} \geq C_{n}-\varepsilon
$$


Combining Proposition 7.3 and Theorem 7.4, with $\mathcal{U}_{n}:=\left(\mathcal{U}, \nu_{n}\right)$ playing the role of $\mathcal{I}_{n}$ in Theorem 7.4 and $C_{n}=n$, we accomplish the task of this section:

COROLlary 7.5. The symmetric Banach ideal $\mathcal{M}=\left(\left[{ }^{n} \mathcal{U}_{n}\right]\right)_{n=1}^{\infty}$ of multilinear mappings is such that:

(a) $A 1 \in \mathcal{M}\left(E_{1}, \ldots, E_{n} ; F\right)$ whenever $A \in \mathcal{M}\left(E_{1}, \ldots, E_{n}, \mathbb{K} ; F\right)$.

(b) $\mathcal{M}$ does not have property (B).

8. Polynomial property (B). In this section we define and explore the polynomial counterpart of property (B).

Definition 8.1. Let $\mathcal{R}$ be a class of continuous homogeneous polynomials between Banach spaces such that for all $n \in \mathbb{N}$ and Banach spaces $E$ and $F$, the component $\mathcal{R}\left({ }^{n} E ; F\right):=\mathcal{P}\left({ }^{n} E ; F\right) \cap \mathcal{R}$ is a linear subspace of $\mathcal{P}\left({ }^{n} E ; F\right)$ endowed with a quasi-norm $\|\cdot\|_{\mathcal{R}}$. As before, $\mathcal{R}\left({ }^{0} E ; F\right)=F$ for every $E$ and $F$. We say that $\mathcal{R}$ has property (B) if there is $C \geq 1$ such that for all $n \in \mathbb{N}, P \in \mathcal{R}\left({ }^{n} E ; F\right)$ and $a \in E$, we have $P a \in \mathcal{R}\left({ }^{n-1} E ; F\right)$ and $\|P a\|_{\mathcal{R}} \leq C\|P\|_{\mathcal{R}}\|a\|$ (remember that $\left.P a(x)=\check{P}\left(a, x^{n-1}\right)\right)$.

From the proof of Theorem 3.2 we have the following result:

Proposition 8.2. Every class of homogeneous polynomials with property (B) with constant $C$ such that the components are quasi-Banach spaces is a global quasi-holomorphy type (global holomorphy type if the components are Banach spaces) with constant $\sigma=2 C$.

Now we illustrate the polynomial property (B). We denote by $\mathcal{L}_{\mathbb{K}}$ and $\mathcal{P}_{\mathbb{K}}$ the classes of all continuous multilinear mappings and homogeneous polynomials between Banach spaces over $\mathbb{K}$ with the usual sup norm, respectively.

EXAMPLE 8.3. Let $\mathcal{H}_{\mathbb{K}}$ be the subclass of $\mathcal{P}_{\mathbb{K}}$ formed by all continuous homogeneous polynomials defined on Hilbert spaces, that is: given $P \in$ $\mathcal{P}_{\mathbb{K}}\left({ }^{n} E ; F\right), P \in \mathcal{H}_{\mathbb{K}}\left({ }^{n} E ; F\right)$ if and only if $E$ is a Hilbert space over $\mathbb{K}$ or $P=0$. From [12, Proposition 1.44] we know that $\|P\|=\|\check{P}\|$ for all scalarvalued polynomials $P$ in $\mathcal{H}_{\mathbb{K}}$. By composing with a given linear functional and applying the Hahn-Banach theorem, we see that $\|P\|=\|\check{P}\|$ for all $P$ in $\mathcal{H}_{\mathbb{K}}$, proving that $\mathcal{H}_{\mathbb{K}}$ has property (B) with respect to the sup norm with constant $C=1$. In particular, $\mathcal{H}_{\mathbb{K}}$ is a global holomorphy type with constant $\sigma=2$ (Proposition 8.2) which is not an ideal of polynomials (obvious).

It is obvious that $\mathcal{L}_{\mathbb{R}}$ and $\mathcal{L}_{\mathbb{C}}$ have property (B) with constant $C=1$. From the classical estimates

$$
\|\check{P}\| \leq \frac{n^{n}}{n !}\|P\| \leq e^{n}\|P\|
$$


which hold for every natural $n$, any Banach spaces $E$ and $F$, and $P \in$ $\mathcal{P}\left({ }^{n} E ; F\right)$, we know that $\mathcal{P}_{\mathbb{R}}$ and $\mathcal{P}_{\mathbb{C}}$ are global holomorphy types with constant $2 e$. We are about to see that the polynomial property (B) works out well in the complex case, but, surprisingly, in the real case it does not.

Proposition 8.4. Let $\mathcal{Q}$ be a closed ideal of polynomials between complex Banach spaces. The following assertions are equivalent.

(a) $\mathcal{Q}$ has property (B) (with best constant $C=e$ ).

(b) $\mathcal{Q}$ is a global holomorphy type (with best constant $\sigma \leq 2 e$ ).

(c) For all $n, E$ and $F, P a \in \mathcal{Q}\left({ }^{n-1} E ; F\right)$ whenever $P \in \mathcal{Q}\left({ }^{n} E ; F\right)$ and $a \in E$.

Proof. (a) $\Rightarrow$ (b). This is a particular case of Proposition 8.2.

(b) $\Rightarrow\left(\right.$ c). Since $P a=(1 / n !) \widehat{d}^{n-1} P(a)$, this implication follows immediately from the definition of global holomorphy type.

(c) $\Rightarrow($ a). The case $n=1$ is immediate. Given $n>1$, complex Banach spaces $E$ and $F, a \in E$ and $P \in \mathcal{Q}\left({ }^{n} E ; F\right)$, by assumption we have $P a \in$ $\mathcal{Q}\left({ }^{n-1} E ; F\right)$. It follows from the definitions that

$$
\|a\| P \frac{a}{\|a\|}=P a=\frac{1}{n !} \widehat{d}^{n-1} P(a) .
$$

Using [16, Corollary 3] with $k=n-1$ we obtain

$$
\begin{aligned}
\|P a\| & =\left\|P \frac{a}{\|a\|}\right\|\|a\|=\frac{1}{n !}\left\|\widehat{d}^{n-1} P\left(\frac{a}{\|a\|}\right)\right\|\|a\| \leq \frac{1}{n !} \frac{n^{n}(n-1) !}{(n-1)^{n-1}}\|P\|\|a\| \\
& =\left(\frac{n}{n-1}\right)^{n-1}\|P\|\|a\| \leq e\|P\|\|a\|,
\end{aligned}
$$

proving that $\mathcal{Q}$ has property (B).

For each $n \in \mathbb{N}$, let $P_{n}$ be the $n$th Nachbin polynomial, that is

$$
P_{n}:\left(\mathbb{C}^{n},\|\cdot\|_{\ell_{1}}\right) \rightarrow \mathbb{C}, \quad P_{n}\left(\left(\lambda_{1}, \ldots, \lambda_{n}\right)\right)=\lambda_{1} \cdots \lambda_{n} .
$$

Since every ideal of polynomials contains the polynomials of finite type and continuous homogeneous polynomials on finite-dimensional spaces are of finite type, each $P_{n}$ belongs to $\mathcal{Q}$. Taking $a_{n}=(1,0, \ldots, 0) \in \mathbb{C}^{n}$, it is easy to see that $\left\|P_{n} a_{n}\right\|=1 / n(n-1)^{n-1}$. But $\left\|P_{n}\right\|=1 / n^{n}$, so

$$
\frac{\left\|P_{n} a_{n}\right\|}{\left\|P_{n}\right\|\left\|a_{n}\right\|}=\frac{n^{n-1}}{(n-1)^{n-1}} \rightarrow e \quad \text { as } n \rightarrow \infty
$$

showing that $e$ is the best constant.

Proposition 8.5. Every closed ideal of polynomials between real Banach spaces lacks property (B).

Proof. Suppose that a closed ideal $\mathcal{Q}$ of polynomials between real Banach spaces has property (B) with constant $C$. For $n \in \mathbb{N}$ and $0 \leq k \leq n$, let 
$c_{n, k}$ be the numbers defined by L. Harris [16, p. 477]. By [16, Theorem 2], for each $n \in \mathbb{N}$ there are $P_{n} \in \mathcal{P}\left({ }^{n}\left(\mathbb{R}^{2},\|\cdot\|_{\ell_{1}}\right)\right)$ and $0 \neq a_{n} \in \mathbb{R}^{2}$ such that $\left\|\widehat{d}^{n-1} P_{n}\left(a_{n}\right)\right\|=c_{n, n-1}\left\|P_{n}\right\|\left\|a_{n}\right\|$. Using again the facts that every ideal of polynomials contains the polynomials of finite type and that continuous homogeneous polynomials on finite-dimensional spaces are of finite type, we deduce that each $P_{n}$ belongs to $\mathcal{Q}$. By [26, Theorem 1], there is an absolute constant $c_{1}>0$ such that $c_{1} n \log n \leq c_{n, 1}$ for all $n$. So,

$$
\begin{aligned}
c_{1} n ! \log n\left\|P_{n}\right\|\left\|a_{n}\right\| & =(n-1) ! c_{1} n \log n\left\|P_{n}\right\|\left\|a_{n}\right\| \leq(n-1) ! c_{n, 1}\left\|P_{n}\right\|\left\|a_{n}\right\| \\
& =c_{n, n-1}\left\|P_{n}\right\|\left\|a_{n}\right\|=\left\|\widehat{d}^{n-1} P_{n}\left(a_{n}\right)\right\|=n !\left\|P_{n} a_{n}\right\| \\
& \leq n ! C\left\|P_{n}\right\|\left\|a_{n}\right\|,
\end{aligned}
$$

yielding the contradiction $c_{1} \log n \leq C$ for all $n$. Hence every closed ideal of polynomials between real Banach spaces fails to have property (B).

Remark 8.6. (a) By Proposition 8.5, $\mathcal{P}_{\mathbb{R}}$, or any closed real ideal of polynomials $\mathcal{Q}$ such that $P a \in \mathcal{Q}\left({ }^{n-1} E ; F\right)$ whenever $P \in \mathcal{Q}\left({ }^{n} E ; F\right)$ and $a \in E$, shows that the converse of Proposition 8.2 fails even for ideals of polynomials, and that property (B) is more appropriate for ideals of multilinear mappings than for ideals of polynomials.

(b) The proofs of Propositions 8.4 and 8.5 use neither property 1.3(ii) (just the containment of the polynomials of finite type) nor the fact that each $\mathcal{Q}\left({ }^{n} E ; F\right)$ is closed in $\mathcal{P}\left({ }^{n} E ; F\right)$ (just the fact that the underlying norm is the usual sup norm).

(c) Let $\mathcal{R}$ be a subclass of $\mathcal{P}_{\mathbb{R}}$ such that each component $\mathcal{R}\left({ }^{n} E ; F\right)$ is a closed subspace of $\mathcal{P}\left({ }^{n} E ; F\right)$. If $\mathcal{R}$ has property (B) with respect to the sup norm, then $\mathcal{R}$ is a global holomorphy type (Proposition 8.2 ) which is not an ideal of polynomials (Proposition 8.5).

9. Everywhere absolutely summing mappings. Several classes have already been studied as multilinear/polynomial generalizations of the ideals of $p$-summing and $(p ; q)$-summing linear operators. Quite promising is the class of all multilinear mappings/polynomials which are absolutely summing at every point of the domain, which we define next. This class was introduced by M. Matos [17] and developed in [21, 22, 23].

Given $p \in[1, \infty)$, let $\ell_{p}(E)$ be the Banach space of all absolutely $p$ summable sequences $\left(x_{j}\right)_{j=1}^{\infty}$ in $E$ with the norm

$$
\left\|\left(x_{j}\right)_{j=1}^{\infty}\right\|_{p}=\left(\sum_{j=1}^{\infty}\left\|x_{j}\right\|^{p}\right)^{1 / p} .
$$

We denote by $\ell_{p}^{w}(E)$ the Banach space of all weakly $p$-summable sequences $\left(x_{j}\right)_{j=1}^{\infty}$ in $E$ with the norm $\left\|\left(x_{j}\right)_{j=1}^{\infty}\right\|_{w, p}=\sup _{\varphi \in B_{E^{\prime}}}\left\|\left(\varphi\left(x_{j}\right)\right)_{j=1}^{\infty}\right\|_{p}$. We denote by $\ell_{p}^{u}(E)$ the closed subspace of $\ell_{p}^{w}(E)$ formed by all sequences 
$\left(x_{j}\right)_{j=1}^{\infty} \in \ell_{p}^{w}(E)$ such that $\lim _{m \rightarrow \infty}\left\|\left(x_{j}\right)_{j=m}^{\infty}\right\|_{w, p}=0$. If $0<p<1$ we have $p$-norms instead of norms, and the resulting spaces are complete metrizable topological vector spaces.

Definition 9.1. Let $1 \leq q \leq p$. An $n$-linear mapping $A \in \mathcal{L}\left(E_{1}, \ldots\right.$, $\left.E_{n} ; F\right)$ is said to be $(p ; q)$-summing at $\left(a_{1}, \ldots, a_{n}\right) \in E_{1} \times \cdots \times E_{n}$ if

$$
\left(A\left(a_{1}+x_{j}^{(1)}, \ldots, a_{n}+x_{j}^{(n)}\right)-A\left(a_{1}, \ldots, a_{n}\right)\right)_{j=1}^{\infty} \in \ell_{p}(F),
$$

whenever $\left(x_{j}^{(k)}\right)_{j=1}^{\infty} \in \ell_{q}^{u}\left(E_{k}\right), k=1, \ldots, n$. The space of all $n$-linear mappings in $\mathcal{L}\left(E_{1}, \ldots, E_{n} ; F\right)$ which are $(p ; q)$-summing at every point of $E_{1} \times$ $\cdots \times E_{n}$ will be denoted by $\mathcal{L}_{\mathrm{as}(p ; q)}^{\mathrm{ev}}\left(E_{1}, \ldots, E_{n} ; F\right)$.

To endow this space with a norm we adapt [17, Section 7]. First we fix some terminology. An $n$-linear mapping $A \in \mathcal{L}\left(E_{1}, \ldots, E_{n} ; F\right)$ is said to be $(p ; q)$-summing if it is $(p ; q)$-summing at $(0, \ldots, 0)$. In this case we write $A \in \mathcal{L}_{\text {as }(p ; q)}\left(E_{1}, \ldots, E_{n} ; F\right)$. It is well known [2, Theorem 1.2(ii)] that $A$ is $(p ; q)$-summing if and only if there is $C \geq 0$ such that for every $m \in \mathbb{N}$ and $x_{j}^{(k)} \in E_{k}, j=1, \ldots, m, k=1, \ldots, n$,

$$
\left\|\left(A\left(x_{j}^{(1)}, \ldots, x_{j}^{(n)}\right)\right)_{j=1}^{m}\right\|_{p} \leq C \prod_{k=1}^{n}\left\|\left(x_{j}^{(k)}\right)_{j=1}^{m}\right\|_{w, q} .
$$

The least such $C$ is denoted by $\|A\|_{\mathrm{as}(p ; q)}$ and it defines an ideal norm on $\mathcal{L}_{\text {as }(p ; q)}\left(E_{1}, \ldots, E_{n} ; F\right)$.

Lemma 9.2. If $A \in \mathcal{L}_{\mathrm{as}(p ; q)}^{\mathrm{ev}}\left(E_{1}, \ldots, E_{n} ; F\right)$ and $\left(a_{1}, \ldots, a_{n}\right) \in E_{1} \times \cdots$ $\times E_{n}$, then there exists a constant $C_{a_{1}, \ldots, a_{n}} \geq 0$ so that

$$
\sum_{j=1}^{\infty}\left\|A\left(a_{1}+x_{j}^{(1)}, \ldots, a_{n}+x_{j}^{(n)}\right)-A\left(a_{1}, \ldots, a_{n}\right)\right\|^{p} \leq C_{a_{1}, \ldots, a_{n}}
$$

whenever $\left\|\left(x_{j}^{(k)}\right)_{j=1}^{\infty}\right\|_{w, q} \leq 1, k=1, \ldots, n$.

Proof. We argue for $n=2$. Let $A \in \mathcal{L}_{\text {as }(p ; q)}^{\mathrm{ev}}\left(E_{1}, E_{2} ; F\right),(a, b) \in E_{1} \times E_{2}$, $\left(x_{j}\right)_{j=1}^{\infty} \in B_{\ell_{q}^{u}\left(E_{1}\right)}$ and $\left(y_{j}\right)_{j=1}^{\infty} \in B_{\ell_{q}^{u}\left(E_{2}\right)}$. It is clear that the linear operators $A(a, \cdot)$ and $A(\cdot, b)$ and the bilinear mapping $A$ are $(p ; q)$-summing. So,

$$
\begin{aligned}
& \left(\sum_{j=1}^{\infty}\left\|A\left(a+x_{j}, b+y_{j}\right)-A(a, b)\right\|^{p}\right)^{1 / p} \\
& \quad \leq\left(\sum_{j=1}^{\infty}\left\|A\left(a, y_{j}\right)\right\|^{p}\right)^{1 / p}+\left(\sum_{j=1}^{\infty}\left\|A\left(x_{j}, b\right)\right\|^{p}\right)^{1 / p}+\left(\sum_{j=1}^{\infty}\left\|A\left(x_{j}, y_{j}\right)\right\|^{p}\right)^{1 / p} \\
& \quad \leq\|A(a, \cdot)\|_{\operatorname{as}(p ; q)}+\|A(\cdot, b)\|_{\operatorname{as}(p ; q)}+\|A\|_{\operatorname{as}(p ; q)}=: C_{a, b}^{1 / p} .
\end{aligned}
$$


Given $A \in \mathcal{L}_{\mathrm{as}(p ; q)}^{\mathrm{ev}}\left(E_{1}, \ldots, E_{n} ; F\right)$, the $n$-linear mapping $\psi_{p, q}(A): \ell_{q}^{u}\left(E_{1}\right)$ $\times \cdots \times \ell_{q}^{u}\left(E_{n}\right) \rightarrow \ell_{p}(F)$ given by

$$
\begin{aligned}
& \psi_{p, q}(A)\left(\left(x_{j}^{(1)}\right)_{j=1}^{\infty}, \ldots,\left(x_{j}^{(n)}\right)_{j=1}^{\infty}\right) \\
& =\left(A\left(x_{1}^{(1)}, \ldots, x_{1}^{(n)}\right),\left(A\left(x_{1}^{(1)}+x_{j}^{(1)}, \ldots, x_{1}^{(n)}+x_{j}^{(n)}\right)-A\left(x_{1}^{(1)}, \ldots, x_{1}^{(n)}\right)\right)_{j=2}^{\infty}\right)
\end{aligned}
$$

is clearly well defined. Actually, much more is true:

Proposition 9.3. Let $A \in \mathcal{L}\left(E_{1}, \ldots, E_{n} ; F\right)$. The following assertions are equivalent:

(a) $A \in \mathcal{L}_{\text {as }(p ; q)}^{\mathrm{ev}}\left(E_{1}, \ldots, E_{n} ; F\right)$.

(b) $\psi_{p, q}(A)$ is well defined and continuous on $\ell_{q}^{u}\left(E_{1}\right) \times \cdots \times \ell_{q}^{u}\left(E_{n}\right)$.

(c) There is $C>0$ such that

$$
\begin{aligned}
&\left(\left\|A\left(a_{1}, \ldots, a_{n}\right)\right\|^{p}+\sum_{j=1}^{\infty} \|\right.\left.A\left(a_{1}+x_{j}^{(1)}, \ldots, a_{n}+x_{j}^{(n)}\right)-A\left(a_{1}, \ldots, a_{n}\right) \|^{p}\right)^{1 / p} \\
& \leq C\left\|\left(a_{1},\left(x_{j}^{(1)}\right)_{j=1}^{\infty}\right)\right\|_{w, q} \cdots\left\|\left(a_{n},\left(x_{j}^{(n)}\right)_{j=1}^{\infty}\right)\right\|_{w, q}
\end{aligned}
$$

for every $\left(a_{1}, \ldots, a_{n}\right) \in E_{1} \times \cdots \times E_{n}$ and $\left(x_{j}^{(k)}\right)_{j=1}^{\infty} \in \ell_{q}^{u}\left(E_{k}\right), k=$ $1, \ldots, n$.

(d) There is $C>0$ such that for every $\left(a_{1}, \ldots, a_{n}\right) \in E_{1} \times \cdots \times E_{n}$, $m \in \mathbb{N}$ and $x_{1}^{(k)}, \ldots, x_{m}^{(k)} \in E_{k}, k=1, \ldots, n$,

$$
\begin{aligned}
&\left(\left\|A\left(a_{1}, \ldots, a_{n}\right)\right\|^{p}+\sum_{j=1}^{m} \|\right.\left.A\left(a_{1}+x_{j}^{(1)}, \ldots, a_{n}+x_{j}^{(n)}\right)-A\left(a_{1}, \ldots, a_{n}\right) \|^{p}\right)^{1 / p} \\
& \leq C\left\|\left(a_{1},\left(x_{j}^{(1)}\right)_{j=1}^{m}\right)\right\|_{w, q} \cdots\left\|\left(a_{n},\left(x_{j}^{(n)}\right)_{j=1}^{m}\right)\right\|_{w, q} .
\end{aligned}
$$

(e) There is $C>0$ such that

$$
\begin{aligned}
&\left(\left\|A\left(a_{1}, \ldots, a_{n}\right)\right\|^{p}+\sum_{j=1}^{\infty} \|\right.\left.A\left(a_{1}+x_{j}^{(1)}, \ldots, a_{n}+x_{j}^{(n)}\right)-A\left(a_{1}, \ldots, a_{n}\right) \|^{p}\right)^{1 / p} \\
& \leq C\left\|\left(a_{1},\left(x_{j}^{(1)}\right)_{j=1}^{\infty}\right)\right\|_{w, q} \cdots\left\|\left(a_{n},\left(x_{j}^{(n)}\right)_{j=1}^{\infty}\right)\right\|_{w, q},
\end{aligned}
$$

for every $\left(a_{1}, \ldots, a_{n}\right) \in E_{1} \times \cdots \times E_{n}$ and $\left(x_{j}^{(k)}\right)_{j=1}^{\infty} \in \ell_{q}^{w}\left(E_{k}\right), k=$ $1, \ldots, n$.

Proof. (a) $\Rightarrow(\mathrm{b})$. Given $k \in \mathbb{N}$ and $\left(x_{j}^{(r)}\right)_{j=1}^{\infty} \in \ell_{q}^{u}\left(E_{r}\right), r=1, \ldots, n$, define

$$
\begin{aligned}
F_{k,\left(x_{j}^{(1)}\right)_{j=1}^{\infty}, \ldots,\left(x_{j}^{(n)}\right)_{j=1}^{\infty}=} & \left\{\left(b_{1}, \ldots, b_{n}\right) \in E_{1} \times \cdots \times E_{n}:\right. \\
& \left.\left\|\psi_{p, q}(A)\left(\left(b_{1},\left(x_{j}^{(1)}\right)_{j=1}^{\infty}\right), \ldots,\left(b_{n},\left(x_{j}^{(n)}\right)_{j=1}^{\infty}\right)\right)\right\|_{p} \leq k\right\} .
\end{aligned}
$$

For each $k \in \mathbb{N}$ and $\left(x_{j}^{(r)}\right)_{j=1}^{\infty} \in B_{\ell_{q}^{u}\left(E_{r}\right)}, r=1, \ldots, n$, this set is closed in $E_{1} \times$ $\cdots \times E_{n}$. Hence so is $F_{k}:=\bigcap F_{k,\left(x_{j}^{(1)}\right)_{j=1}^{\infty}, \ldots,\left(x_{j}^{(n)}\right)_{j=1}^{\infty}}$, where the intersection 
is taken over all $\left(x_{j}^{(r)}\right)_{j=1}^{\infty} \in B_{\ell_{q}^{u}\left(E_{r}\right)}, r=1, \ldots, n$. Using Lemma 9.2 it is not difficult to check that $E_{1} \times \cdots \times E_{n}=\bigcup_{k \in \mathbb{N}} F_{k}$. By the Baire category theorem there is $k_{0}$ such that $F_{k_{0}}$ has non-empty interior. Let $\left(b_{1}, \ldots, b_{n}\right)$ be in the interior of $F_{k_{0}}$, so there is $0<\varepsilon<1$ such that

$$
\left\|\psi_{p, q}(A)\left(\left(c_{1},\left(x_{j}^{(1)}\right)_{j=1}^{\infty}\right), \ldots,\left(c_{n},\left(x_{j}^{(n)}\right)_{j=1}^{\infty}\right)\right)\right\|_{p} \leq k_{0}
$$

whenever $\left\|c_{r}-b_{r}\right\|<\varepsilon$ and $\left(x_{j}^{(r)}\right)_{j=1}^{\infty} \in B_{\ell_{q}^{u}\left(E_{r}\right)}, r=1, \ldots, n$. Thus, if $\left(x_{j}^{(r)}\right)_{j=1}^{\infty} \in \ell_{q}^{u}\left(E_{r}\right), r=1, \ldots, n$, are such that $\left\|\left(x_{j}^{(r)}\right)_{j=1}^{\infty}\right\|_{w, q}<\varepsilon$, then $\left\|\left(b_{r}+x_{1}^{(r)}\right)-b_{r}\right\|<\varepsilon$ and $\left(x_{j}^{(r)}\right)_{j=2}^{\infty} \in B_{\ell_{q}^{u}\left(E_{r}\right)}, r=1, \ldots, n$. It follows that

$$
\left\|\psi_{p, q}(A)\left(\left(b_{1}, 0,0, \ldots\right)+\left(x_{j}^{(1)}\right)_{j=1}^{\infty}, \ldots,\left(b_{n}, 0,0, \ldots\right)+\left(x_{j}^{(n)}\right)_{j=1}^{\infty}\right)\right\|_{p} \leq k_{0} .
$$

Therefore, $\psi_{p, q}(A)$ is bounded in the ball of radius $\varepsilon$ and center at the point $\left(\left(b_{1}, 0,0, \ldots\right), \ldots,\left(b_{n}, 0,0, \ldots\right)\right) \in \ell_{q}^{u}\left(E_{1}\right) \times \cdots \times \ell_{q}^{u}\left(E_{n}\right)$. It follows that $\psi_{p, q}(A)$ is continuous.

To prove $(\mathrm{b}) \Rightarrow(\mathrm{c})$, observe that

$$
\begin{aligned}
& \left\|\psi_{p, q}(A)\left(\left(a_{1},\left(x_{j}^{(1)}\right)_{j=1}^{\infty}\right), \ldots,\left(a_{n},\left(x_{j}^{(n)}\right)_{j=1}^{\infty}\right)\right)\right\|^{p} \\
& \quad=\left\|A\left(a_{1}, \ldots, a_{n}\right)\right\|^{p}+\sum_{j=1}^{\infty}\left\|A\left(a_{1}+x_{j}^{(1)}, \ldots, a_{n}+x_{j}^{(n)}\right)-A\left(a_{1}, \ldots, a_{n}\right)\right\|^{p},
\end{aligned}
$$

and put $C=\left\|\psi_{p, q}(A)\right\|$. The implications $(\mathrm{c}) \Rightarrow(\mathrm{d}) \Rightarrow(\mathrm{e}) \Rightarrow($ a) are trivial.

Proposition 9.4. The map

$$
\psi_{p, q}: \mathcal{L}_{\mathrm{as}(p ; q)}^{\mathrm{ev}}\left(E_{1}, \ldots, E_{n} ; F\right) \rightarrow \mathcal{L}\left(\ell_{q}^{u}\left(E_{1}\right), \ldots, \ell_{q}^{u}\left(E_{n}\right) ; \ell_{p}(F)\right)
$$

is linear injective and its range is closed in $\mathcal{L}\left(\ell_{q}^{u}\left(E_{1}\right), \ldots, \ell_{q}^{u}\left(E_{n}\right) ; \ell_{p}(F)\right)$.

Proof. $\psi_{p, q}$ is well defined by Proposition 9.3. Its linearity and injectivity are obvious. Let us prove that its range is closed. Let $\left(A_{k}\right)_{k=1}^{\infty} \subset$ $\mathcal{L}_{\text {as }(p ; q)}^{\text {ev }}\left(E_{1}, \ldots, E_{n} ; F\right)$ with $\psi_{p, q}\left(A_{k}\right) \rightarrow h$. So, $\left\|\psi_{p, q}\left(A_{k}\right)\right\| \rightarrow\|h\|$. Given $a_{j} \in E_{j}, j=1, \ldots, n$, from

$$
\psi_{p, q}\left(A_{k}\right)\left(\left(a_{1}, 0,0, \ldots\right), \ldots,\left(a_{n}, 0,0, \ldots\right)\right)=\left(A_{k}\left(a_{1}, \ldots, a_{n}\right), 0, \ldots, 0\right),
$$

if $\pi_{j}$ denotes the projection onto the $j$ th coordinate, we get

$$
\lim _{k} A_{k}\left(a_{1}, \ldots, a_{n}\right)=\pi_{1}\left(h\left(\left(a_{1}, 0,0, \ldots,\right), \ldots,\left(a_{n}, 0,0, \ldots,\right)\right)\right) .
$$

So we can define $A \in \mathcal{L}\left(E_{1}, \ldots, E_{n} ; F\right)$ by $A\left(a_{1}, \ldots, a_{n}\right):=\lim _{k} A_{k}\left(a_{1}, \ldots, a_{n}\right)$. Let $\left(b_{1}, \ldots, b_{n}\right) \in E_{1} \times \cdots \times E_{n}$ and $\left(x_{j}^{(r)}\right)_{j=1}^{\infty} \in \ell_{q}^{u}\left(E_{r}\right), r=1, \ldots, n$. For each $m \in \mathbb{N}$, 


$$
\begin{aligned}
& \left\|\left(A\left(b_{1}+x_{j}^{(1)}, \ldots, b_{n}+x_{j}^{(n)}\right)-A\left(b_{1}, \ldots, b_{n}\right)\right)_{j=1}^{m}\right\|_{p} \\
& \quad \leq\left(\left\|A\left(b_{1}, \ldots, b_{n}\right)\right\|^{p}+\sum_{j=1}^{m}\left\|A\left(b_{1}+x_{j}^{(1)}, \ldots, b_{n}+x_{j}^{(n)}\right)-A\left(b_{1}, \ldots, b_{n}\right)\right\|^{p}\right)^{1 / p} \\
& \quad=\lim _{k}\left\|\psi_{p, q}\left(A_{k}\right)\left(\left(b_{1},\left(x_{j}^{(1)}\right)_{j=1}^{m}\right), \ldots,\left(b_{n},\left(x_{j}^{(n)}\right)_{j=1}^{m}\right)\right)\right\|_{p} \\
& \quad \leq \lim _{k}\left\|\psi_{p, q}\left(A_{k}\right)\right\| \prod_{r=1}^{n}\left\|\left(b_{r},\left(x_{j}^{(r)}\right)_{j=1}^{m}\right)\right\|_{w, q} \\
& \quad \leq\|h\| \prod_{r=1}^{n}\left(\left\|b_{r}\right\|+\left\|\left(x_{j}^{(r)}\right)_{j=1}^{m}\right\|_{w, q}\right) \leq\|h\| \prod_{r=1}^{n}\left(\left\|b_{r}\right\|+\left\|\left(x_{j}^{(r)}\right)_{j=1}^{\infty}\right\|_{w, q}\right),
\end{aligned}
$$

showing that $A \in \mathcal{L}_{\mathrm{as}(p ; q)}^{\mathrm{ev}}\left(E_{1}, \ldots, E_{n} ; F\right)$. Moreover, it is not difficult to see that

$$
\begin{aligned}
\pi_{j}\left(h \left(\left(x_{i}^{(1)}\right)_{i=1}^{\infty}, \ldots\right.\right. & \left.\left.,\left(x_{i}^{(n)}\right)_{i=1}^{\infty}\right)\right) \\
& =A\left(x_{1}^{(1)}+x_{j}^{(1)}, \ldots, x_{1}^{(n)}+x_{j}^{(n)}\right)-A\left(x_{1}^{(1)}, \ldots, x_{1}^{(n)}\right)
\end{aligned}
$$

for every $j=2,3, \ldots$ Since

$$
\begin{aligned}
\pi_{1}\left(h\left(\left(x_{i}^{(1)}\right)_{i=1}^{\infty}, \ldots,\left(x_{i}^{(n)}\right)_{i=1}^{\infty}\right)\right) & =A\left(x_{1}^{(1)}, \ldots, x_{1}^{(n)}\right), \\
h\left(\left(x_{i}^{(1)}\right)_{i=1}^{\infty}, \ldots,\left(x_{i}^{(n)}\right)_{i=1}^{\infty}\right) & =\psi_{p, q}(A)\left(\left(x_{i}^{(1)}\right)_{i=1}^{\infty}, \ldots,\left(x_{i}^{(n)}\right)_{i=1}^{\infty}\right) .
\end{aligned}
$$

So $\psi_{p, q}(A)=h$, and therefore $h$ belongs to the range of $\psi_{p, q}$.

It is plain that $\mathcal{L}_{\mathrm{as}(p ; q)}^{\mathrm{ev}}$ is an ideal of multilinear mappings. From Propositions 9.3 and 9.4 it follows that the correspondence

$$
A \in \mathcal{L}_{\mathrm{as}(p ; q)}^{\mathrm{ev}}\left(E_{1}, \ldots, E_{n} ; F\right) \mapsto\|A\|_{\mathrm{ev}(p ; q)}:=\left\|\psi_{p, q}(A)\right\|
$$

makes $\mathcal{L}_{\text {as }(p ; q)}^{\mathrm{ev}}\left(E_{1}, \ldots, E_{n} ; F\right)$ a Banach space. Straightforward computations show that $\left(\mathcal{L}_{\mathrm{as}(p ; q)}^{\mathrm{ev}},\|\cdot\|_{\mathrm{ev}(p ; q)}\right)$ is a Banach ideal of multilinear mappings modulo property $1.3\left(\mathrm{ii}^{\prime}\right)$. For every $A \in \mathcal{L}_{\text {as }(p ; q)}^{\mathrm{ev}}\left(E_{1}, \ldots, E_{n} ; F\right),\|A\|_{\mathrm{ev}(p ; q)}$ is the least constant $C$ for which the inequalities in Proposition 9.3 always hold.

Definition 9.5 (M. Matos [17]). Let $1 \leq q \leq p$. An $n$-homogeneous polynomial $P \in \mathcal{P}\left({ }^{n} E ; F\right)$ is said to be absolutely $(p ; q)$-summing (or $(p ; q)$ summing ) at $a \in E$ if $\left(P\left(a+x_{j}\right)-P(a)\right)_{j=1}^{\infty} \in \ell_{p}(F)$ whenever $\left(x_{j}\right)_{j=1}^{\infty} \in$ $\ell_{q}^{u}(E)$. The space of all $n$-homogeneous polynomials in $\mathcal{P}\left({ }^{n} E ; F\right)$ which are $(p ; q)$-summing at every point of $E$ will be denoted by $\mathcal{P}_{\text {as }(p ; q)}^{\text {ev }}\left({ }^{n} E ; F\right)$.

We let $P \in \mathcal{P}_{\text {as }(p ; q)}^{\text {ev }}\left({ }^{n} E ; F\right) \mapsto\|P\|_{\text {ev }(p ; q)}$ be the norm on $\mathcal{P}_{\text {as }(p ; q)}^{\text {ev }}\left({ }^{n} E ; F\right)$ introduced in [17]. Adapting the proof of [21, Proposition 4] we find that $P \in \mathcal{P}_{\text {as }(p ; q)}^{\mathrm{ev}}\left({ }^{n} E ; F\right)$ if and only if $\check{P} \in \mathcal{L}_{\text {as }(p ; q)}^{\mathrm{ev}}\left({ }^{n} E ; F\right)$. Characterizations analogous to those of Proposition 9.3 also hold for polynomials. In particular, 
$\mathcal{P}_{\text {as }(p ; q)}^{\text {ev }}=\mathcal{P}_{\mathcal{L}_{\text {as }(p ; q)}^{\text {ev }}}$ as ideals. M. Matos [17, Proposition 7.8] proved that $\mathcal{P}_{\text {as }(p ; q)}^{\text {ev }}$ is a global holomorphy type with respect to the norm $P \mapsto\|P\|_{\text {ev }(p ; q)}$ with constant $\sigma=2 e$.

TheOREM 9.6. $\mathcal{L}_{\mathrm{as}(p ; q)}^{\mathrm{ev}}$ has property $(\mathrm{B})$ with constant $C=1$. So, $\mathcal{P}_{\operatorname{as}(p ; q)}^{\mathrm{ev}}$ is a global holomorphy type with respect to the norm $P \mapsto\|\check{P}\|_{\mathrm{ev}(p ; q)}$ with $\sigma=2$.

Proof. The second assertion follows from the first by applying Theorem 3.2 and observing that its proof does not depend on the property that $\mathcal{L}_{\text {as }(p ; q)}^{\mathrm{ev}}$ lacks, namely, $1.3\left(\mathrm{ii}^{\prime}\right)$. Let $A \in \mathcal{L}_{\text {as }(p ; q)}^{\mathrm{ev}}\left(E_{1}, \ldots, E_{n+1} ; F\right)$ and $a \in$ $E_{n+1}$. From

$$
\begin{aligned}
A a\left(a_{1}+x_{j}^{(1)}, \ldots, a_{n}+x_{j}^{(n)}\right)-A a\left(a_{1}, \ldots, a_{n}\right) \\
\quad=A\left(a_{1}+x_{j}^{(1)}, \ldots, a_{n}+x_{j}^{(n)}, a+0\right)-A\left(a_{1}, \ldots, a_{n}, a\right),
\end{aligned}
$$

it is clear that $A a \in \mathcal{L}_{\text {as }(p ; q)}^{\mathrm{ev}}\left(E_{1}, \ldots, E_{n} ; F\right)$. Given $a_{k} \in E_{k}$ and $\left(x_{j}^{(k)}\right)_{j=1}^{\infty} \in$ $\ell_{q}^{u}\left(E_{k}\right), k=1, \ldots, n$, by Proposition 9.3 we get

$$
\begin{aligned}
\left(\left\|A a\left(a_{1}, \ldots, a_{n}\right)\right\|^{p}+\sum_{j=1}^{\infty}\left\|A a\left(a_{1}+x_{j}^{(1)}, \ldots, a_{n}+x_{j}^{(n)}\right)-A a\left(a_{1}, \ldots, a_{n}\right)\right\|^{p}\right)^{1 / p} & \\
= & \left(\left\|A\left(a_{1}, \ldots, a_{n}, a\right)\right\|^{p}\right. \\
& \left.+\sum_{j=1}^{\infty}\left\|A\left(a_{1}+x_{j}^{(1)}, \ldots, a_{n}+x_{j}^{(n)}, a+0\right)-A\left(a_{1}, \ldots, a_{n}, a\right)\right\|^{p}\right)^{1 / p} \\
\leq & \|A\|_{\operatorname{ev}(p ; q)}\|a\|\left\|\left(a_{1},\left(x_{j}^{(1)}\right)_{j=1}^{\infty}\right)\right\|_{w, q} \cdots\left\|\left(a_{n},\left(x_{j}^{(n)}\right)_{j=1}^{\infty}\right)\right\|_{w, q} .
\end{aligned}
$$

It follows that $\|A a\|_{\mathrm{ev}(p ; q)} \leq\|A\|_{\mathrm{ev}(p ; q)}\|a\|$.

\section{References}

[1] R. Alencar and M. Matos, Some classes of multilinear mappings between Banach spaces, Publ. Dep. Analisis Mat. Univ. Complut. Madrid 12 (1989).

[2] G. Botelho, Cotype and absolutely summing multilinear mappings and homogeneous polynomials, Proc. Roy. Irish Acad. Sect. A 97 (1997), 145-153.

[3] -, Ideals of polynomials generated by weakly compact operators, Note Mat. 24 (2005), 69-102.

[4] G. Botelho and D. Pellegrino, Two new properties of ideals of polynomials and applications, Indag. Math. (N.S.) 16 (2005), 157-169.

[5] - - - On symmetric ideals of multilinear mappings between Banach spaces, J. Austr. Math. Soc. 81 (2006), 141-148.

[6] H.-A. Braunss, Ideale multilinearer Abbildungen und Räume holomorpher Funktionen, Dissertation (A), Pädagogische Hochschule 'Karl Liebknecht', Potsdam, 1984.

[7] —, Multi-ideals with special properties, Blätter Potsdamer Forschungen 1/87, Potsdam, 1987. 
[8] H.-A. Braunss and H. Junek, On types of polynomials and holomorphic functions on Banach spaces, Note Mat. 10 (1990), 47-58.

[9] - - - Ideals of polynomials and multilinear mappings, unpublished notes.

[10] J. Diestel, H. Jarchow and A. Tonge, Absolutely Summing Operators, Cambridge Univ. Press, Cambridge, 1995.

[11] S. Dineen, Holomorphy types on a Banach space, Studia Math. 39 (1971), 241-288.

[12] —, Complex Analysis on Infinite Dimensional Spaces, Springer, London, 1999.

[13] K. Floret and D. García, On ideals of polynomials and multilinear mappings between Banach spaces, Arch. Math. (Basel) 81 (2003), 300-308.

[14] S. Geiss, Ein Faktorisierungssatz für multilineare Funktionale, Math. Nachr. 134 (1987), 149-159.

[15] M. González and J. Gutiérrez, Injective factorization of holomorphic mappings, Proc. Amer. Math. Soc. 127 (1999), 1715-1721; Erratum, 129 (2001), 1255-1256.

[16] L. Harris, A Bernstein-Markov theorem for normed spaces, J. Math. Anal. Appl. 208 (1997), 476-486.

[17] M. Matos, Nonlinear absolutely summing mappings, Math. Nachr. 258 (2003), 71-89.

[18] - Mappings between Banach spaces that send mixed summable sequences into absolutely summable sequences, J. Math. Anal. Appl. 297 (2004), 833-851.

[19] L. Nachbin, Topology on Spaces of Holomorphic Mappings, Springer, New York, 1969.

[20] D. Pellegrino, Strongly almost summing holomorphic mappings, J. Math. Anal. Appl. 287 (2003), 244-252.

[21] —, Almost summing mappings, Arch. Math. (Basel) 82 (2004), 68-80.

[22] - Cotype and nonlinear absolutely summing mappings, Math. Proc. Roy. Irish Acad. Sect. A 105 (2005), 75-91.

[23] D. Pellegrino and M. Souza, Fully summing multilinear and holomorphic mappings into Hilbert spaces, Math. Nachr. 278 (2005), 877-887.

[24] A. Pietsch, Operator Ideals, North-Holland, Amsterdam, 1980.

[25] - Ideals of multilinear functionals (designs of a theory), in: Proc. Second Int. Conf. on Operator Algebras, Ideals and Their Applications in Theoretical Physics, Teubner-Texte Math. 67, Leipzig, 1983, 185-199.

[26] S. Révész and Y. Sarantopoulos, On Markov constants of homogeneous polynomials over real normed spaces, East J. Approx. 9 (2003), 277-304.

[27] P. Wojtaszczyk, Banach Spaces for Analysts, Cambridge Univ. Press, Cambridge, 1991.

Faculdade de Matemática

Universidade Federal de Uberlândia

38.400-902 Uberlândia, Brazil

E-mail: botelho@ufu.br

Institute of Mathematics

University of Potsdam

14469 Potsdam Germany

e-mails: braunss@rz.uni-potsdam.de

junek@rz.uni-potsdam.de
Departamento de Matemática e Estatística

Universidade Federal de Campina Grande

Caixa Postal 10044

58.109-970 Campina Grande, Brazil

E-mail: dmp@dme.ufcg.edu.br 